Erschienen in: Kreimer, Margareta/Sturn, Richard/Dujmovits, Rudolf (Hrsg.), Paradigmenwechsel in der Familienpolitik, 2011, Wiesbaden: Springer VS Verlag, S. 55-82.

\title{
Familienpolitische Positionen in Österreich: Fragmente eines Paradigmenwechsels
}

\author{
Rudolf Dujmovits ${ }^{1}$
}

\section{$1 \quad$ Einleitung}

Nachdem sie für längere Zeit eher ein Nischendasein geführt hat, ist die Familienpolitik in Deutschland und Österreich ins Zentrum politischer Auseinandersetzungen gerückt. Wie Sturn in seinem einleitenden Betrag in diesem Band zeigt, resultiert dieser Bedeutungsgewinn auch aus der zunehmenden Erkenntnis über den Zusammenhang familienpolitischer Problemlagen mit großen gesellschaftspolitischen Themen wie der Geschlechtergleichstellung, Mängeln im Bildungswesen, Befürchtungen bezüglich des Verlustes wirtschaftlicher Wettbewerbsfähigkeit, der erwarteten Unfinanzierbarkeit sozialer Sicherungssysteme und des Zerbrechens des gesellschaftlichen Zusammenhalts aufgrund zunehmender kultureller (und anderer) Heterogenität(en), unter anderem infolge von Migration.

Das alles findet seinen Niederschlag in öffentlichen Kontroversen über die ,richtige“ Familienpolitik. Dabei werden traditionelle familienpolitische Zielsetzungen durch neue Teilziele ergänzt, aber auch grundsätzlich in Frage gestellt. Falls es dabei nicht nur um kurzfristig orientierte, die WählerInnenstimmen maximierende politische (Schein)Gefechte geht, sondern auch um eine - infolge veränderter gesellschaftlicher Rahmenbedingungen notwendig werdende - substantielle inhaltliche Auseinandersetzung, ist zu erwarten, dass sich diese Kontroversen auch in den Positionen familienpolitisch relevanter gesellschaftlicher Institutionen sowie in konkreten familienpolitischen Maßnahmen niederschlagen.

Dieser Beitrag untersucht, ob und in welche Richtung sich die familienpolitischen Positionen politischer Parteien und Interessenvertretungen in Österreich in den vergangenen Jahrzehnten verändert haben. Im folgenden Beitrag von Kreimer werden allfällige Veränderungen auf der Ebene der realisierten Maßnahmen analysiert und die Ergebnisse der beiden Beiträge zusammengeführt und diskutiert.

Ausgangs- und Referenzpunkt für diese Analyse ist die modernisierte Variante des traditionellen Familienmodells der männlichen Versorgerehe. In der Urform beruht dieses Modell auf einer vollständigen Spezialisierung der Geschlechter auf Erwerbsarbeit einerseits und reproduktive Familienarbeit andererseits (z. B. Lewis/Ostner 1994). In diesem Modell ist die Hausfrau zuständig für die Betreuungs-, Erziehungs- und Pflegearbeiten, die sich von der Kinderbetreuung über sonstige, der alltäglichen Reproduktion dienende Maßnahmen bis zur Altenpflege erstrecken. Die finanzielle Grundlage für diese familiäre Arbeitsteilung schafft der Ehemann über seine Tätigkeit im Erwerbssystem - male-breadwinner und femalecaretaker.

Idealtypisch muss das politische Ziel im Rahmen dieses Paradigmas darin bestehen, die traditionelle Arbeitsteilung zwischen ernährendem Mann und betreuender Hausfrau zu ermöglichen, beispielsweise indem der Ernährer die familiäre Reproduktion sicher stellende „Familienlöhne“ auf dem Markt verdienen kann. Da dies angesichts der Bedingungen auf den Arbeitsmärkten in kapitalistischen Gesellschaften vielfach nicht möglich ist, die Markteinkommen also zu Familien- und Kinderarmut führen können, hat der Staat subsidiär durch ergänzende familienpolitische Unterstützungen einzuspringen. ${ }^{2}$

Modernisierte Formen des traditionellen male-breadwinner-Modells unterstützen dagegen auch die Beteiligung von Müttern im marktlich organisierten Erwerbssystem, die durch geeignete staatliche Rahmenbedingungen ermöglicht werden soll. Die öffentliche Kinderbetreuung und die damit verbundenen Rechte sind jedoch auch in diesem Modell ebenso wenig umfassend ausgebaut, wie eine eigenständige und existenzsichernde Absicherung der Frauen vorgesehen ist und eine gleichmäßigere innerfamiliäre Aufteilung von unbezahlter Familienarbeit und bezahlter Erwerbsarbeit angestrebt wird. Zudem bleibt die Erwerbsarbeit die primäre ökonomische Grundlage für die Reproduktion der Familie. Die Vereinbarkeit von Erwerbsarbeit und Familie für Mütter ist in diesem Rahmen funktional: Sie soll zum einen die Generierung eines ausreichenden Familieneinkommens ermöglichen und zum anderen

1 Dieser Beitrag entstand in enger Zusammenarbeit mit Margareta Kreimer.

2 Sturn diskutiert in seinem Beitrag in diesem Band diverse Varianten dieses Modells. 
genügend Zeit für die familiäre Kinderbetreuung belassen. Die bestmögliche Ausnutzung des Humanvermögens der Frauen auf dem Arbeitsmarkt spielt in diesem Rahmen keine Rolle. Vielfach läuft dieses Modell auf eine Abfolge nicht existenzsichernder Teilzeitarbeit und Erwerbsunterbrechungen hinaus (z. B. Dingeldey 2000, Leitner 2003, Klenner 2007).

Die Hauptverantwortung für die Erziehung und Bildung der Kinder verbleibt in diesem Modell weiterhin primär im Bereich der Familie. ${ }^{3}$ Die Sozialisierung und Vorbereitung der Kinder auf ihr weiteres Leben soll demnach in erster Linie im Familienverband erfolgen. VertreterInnen solcher Modelle unterstellen letztlich, dass die Familien die dafür erforderlichen Kompetenzen mitbringen und dass diese Form der familiären Arbeitsteilung die optimalen Bedingungen für die gesellschaftliche Reproduktion unter anderem auch in Form ausreichend hoher Geburtenraten bietet. Staatliche Eingriffe in den Bereich der Familie werden grundsätzlich skeptisch beurteilt und nur in besonderen Fällen als notwendig erachtet. Schule und andere Institutionen unterstützen die Familie bei ihrer Erziehungs- und Bildungsaufgabe nur subsidiär. Eine schichtspezifische Stabilisierung des gesellschaftlichen Gefüges und entsprechend selektive gesellschaftliche Aufstiegschancen von Kindern sind eine unvermeidliche (von manchen insgeheim wohl auch gewünschte, wenngleich kaum öffentlich argumentierte $)^{4}$ Folge dieses familienpolitischen Leitbildes.

Für Deutschland wurde kürzlich eine Neuausrichtung der Familienpolitik sowohl auf der Ebene der Maßnahmen als auch der grundsätzlichen Positionen konstatiert (Leitner 2008; Ostner 2008, 2006). ${ }^{5}$ Im Folgenden wird untersucht, ob und in welcher Form sich auch für Österreich eine solche Abkehr von einem modernisierten male-breadwinner-Modell festmachen lässt. Im folgenden Abschnitt werden einige Gründe beschrieben, die für grundlegende Änderungen in der Familienpolitik sprechen. Vor diesem Hintergrund werden im dritten Abschnitt Kriterien zur Beurteilung eines allfälligen Paradigmenwechsels in den familienpolitischen Positionen von Parteien und Verbänden entwickelt. Anschließend werden diese Kriterien auf diverse Parteiprogramme und Positionspapiere aus jüngerer Zeit angewendet. Der Beitrag schließt mit einer zusammenfassenden Einordnung dieser Entwicklung in den letzten 15 Jahren.

\section{Triebkräfte für einen Paradigmenwechsel}

Sind die in jüngster Zeit hoch gekommenen politischen Auseinandersetzungen über die „richtige" Familienpolitik nur von engen und kurzfristigen politischen Interessen getriebene Scharmützel oder werden sie von grundlegenderen, dauerhaft wirkenden Kräften gestützt? Es gibt starke Argumente dafür, dass die Diskussion über eine Neuorientierung der Familienpolitik ursächlich mit großen gesellschaftlichen Problemlagen sowie sich verändernden gesellschaftlichen, wirtschaftlichen und kulturellen Rahmenbedingungen nicht nur verknüpft ist, sondern von diesen auch getrieben wird.

Es herrscht auch Übereinstimmung darüber, dass die Institution des Marktes von sich aus die familiale Reproduktion langfristig nicht in jedem Fall sicherstellen kann. Sowohl die daraus resultierenden Problemlagen - Sturn spricht in diesem Zusammenhang von ,reproduktivem Marktversagen“ - als auch die daraus resultierenden, zur Stützung der Familie erforderlichen Rahmenbedingungen und Maßnahmen sind von den aktuellen Ausformungen der Ausdifferenzierung der Arbeitsteilung in Wirtschaft und Gesellschaft abhängig. Deshalb

\footnotetext{
3 Das gilt auch für den Bereich der Langzeitpflege von älteren und dauerhaft kranken Personen, der in diesem und dem folgenden Beitrag nicht weiter behandelt wird, da wir uns auf die Familienpolitik im engeren Sinn konzentrieren. Es sei aber darauf verwiesen, dass auch die Erbringung der Pflegeleistungen im Familienverband überwiegend durch Frauen erfolgt. Bezüglich der im Folgenden diskutierten familienpolitischen Ziele ergeben sich daraus Problemstellungen, die in vielerlei Hinsicht jenen bei der Kinderbetreuung gleichen, teilweise aber auch deutlich darüber hinausgehen (vgl. beispielsweise Meier/Kreimer 2011).

4 Sarrazin (2010) mit seiner umstrittenen, aber viel diskutierten These, wonach Deutschland letztlich an der unzureichenden Reproduktion mittlerer und vor allem höherer, bildungsnaher Schichten leidet, stellt diesbezüglich eine Ausnahme dar.

5 Dieser Wechsel wurde und wird in hohem Ausmaß mit ökonomischer Sachlogik begründet. An dieser „Ökonomisierung der Lebenswelt“ (Titel des Beitrags von Ostner 2008) und „Entfamilialisierung der Elternschaft“ (Ostner 2008: 188) wird eine zu einseitige Betonung des Aspekts der ökonomischen Nutzung des Humankapitals der Frauen und Kinder und die damit einhergehende Vermarktlichung weiterer Lebensbereiche kritisiert, eine Auseinandersetzung, auf die an dieser Stelle nicht im Detail eingegangen werden kann. Aus österreichischer Perspektive vgl. dazu den Beitrag von Schratzenstaller in diesem Band, die ebenfalls auf die Grenzen einer nur auf ökonomischer Sachlogik beruhenden Argumentation hinweist.
} 
bedarf es immer wieder einer Anpassung familienpolitischer Ziele und Maßnahmen, wobei es auch Freiheitsgrade für eine normative politische Gestaltung gibt.

Die Bedingungen und die daraus resultierenden Problemlagen, unter denen Familien in entwickelten Industriegesellschaften wie Deutschland oder Österreich ihre Reproduktionsaufgaben in den Bereichen Betreuung und Pflege, Erziehung und Bildung zu erbringen haben, haben sich in den vergangenen Jahrzehnten zweifellos verändert. Das lässt sich mit einer Reihe empirisch belegbarer Phänomene zeigen: ${ }^{6}$

\subsection{Instabile und zunehmend prekäre Beschäftigungsverhältnisse der Männer}

Das Risiko von Schwankungen bzw. (temporären) Ausfällen des Markteinkommens steigt bei den Männern aufgrund der Flexibilisierung der Beschäftigungsverhältnisse, des Anstiegs von Niedriglohnbeschäftigungen sowie zunehmenden Diskontinuitäten in den Berufsverläufen. Den Hintergrund dieses Strukturwandels auf den Arbeitsmärkten bilden neben wirtschaftlichen Krisen insbesondere individuell unvorhersehbare Entwertungen des Humankapitals in Folge technologischen Fortschritts bzw. Veränderungen der räumlichen Arbeitsteilung. Auch für männliche Arbeitskräfte gilt daher immer häufiger, dass Phasen der Beschäftigung mit Phasen der Arbeitssuche und -losigkeit bzw. der Requalifikation abwechseln, dass prekäre Beschäftigungsverhältnisse anstelle vollversicherungspflichtiger, vollzeitiger und unbefristeter „Normalarbeitsverhältnisse“ eingegangen werden müssen.?

Die im modernisierten breadwinner-Modell quasi als „Norm“ unterstellte lebenslange, durchgehende Vollzeitbeschäftigung des Mannes wird immer häufiger von der Regel zur Ausnahme. Eine planbare und dauerhafte finanzielle Absicherung von Familien wird dadurch erschwert. Zur Erhaltung der Reproduktionsfunktion der betroffenen Familien ist dieses erhöhte Einkommensrisiko durch geeignete Reaktionen auf individueller Ebene, wie Berufstätigkeit beider Partner, sowie durch geeignete staatliche Maßnahmen abzufedern.

\subsection{Ausbildungsniveau und Erwerbstätigkeit der Frauen steigen}

Die Erwerbsquote ${ }^{8}$ und das Ausbildungsniveau der Frauen ${ }^{9}$ sind in den vergangenen Jahrzehnten deutlich gestiegen. Die gleichzeitige Berufstätigkeit beider Partner sowie die Höherqualifizierung der Frauen können als Versicherung von Familien gegen das zunehmende Einkommensrisiko der Männer auf den Arbeitsmärkten interpretiert werden. Zudem steigt die Erwerbsorientierung insbesondere höher qualifizierter Frauen. Auch seitens der Arbeitgeber wird eine umfassendere Nutzung des Humanvermögens der Frauen gefordert, wie später noch gezeigt wird. Schließlich sind diese Entwicklungen auch auf die berechtigten Forderungen nach Geschlechtergleichstellung und wirtschaftlicher Unabhängigkeit vom Ehemann zurückzuführen.

\subsection{Instabilere und vielfältigere Familienstrukturen}

Nicht nur die Berufsverläufe von Männern und Frauen, sondern auch die Familienstrukturen werden diskontinuierlicher. Die lebenslange Gemeinschaft von Mann und Frau in einer Ehe zeigt deutliche Auflösungstendenzen, wie sich an der Zunahme der Scheidungen, der alleinerziehenden Haushalte, der Patchwork-Familien bis hin zum Wunsch von gleichgeschlechtlichen Paaren nach Kindern zeigen lässt (BMWFJ 2010).

6 Zwischen den im Folgenden dargestellten Phänomenen bestehen fallweise auch Wechselwirkungen, die im Rahmen dieses Beitrags nur fallweise angedeutet, aber nicht systematisch analysiert werden können.

7 Vgl. dazu für Österreich beispielsweise Geisberger/Knittler (2010), für Deutschland Kalina/Weinkopf (2008).

8 Die Erwerbsquote der Frauen in Österreich ist von knapp unter 50\% im Jahr 1974 auf rund 67\% im Jahr 2008 gestiegen (bezogen auf die 15- bis 64-Jährigen); die Erwerbsquote der Männer sank im selben Zeitraum von rund $85 \%$ auf rund $81 \%$.

92008 wiesen rund 13\% der 25- bis 34-jährigen Frauen als höchsten Abschluss nur die Pflichtschule auf, 1981 waren es noch 41,6\%. 2008 hatten 19,6\% der 25- bis 34-jährigen Frauen gegenüber 14,3\% der Männer in der gleichen Altersgruppe einen Tertiär-Abschluss, 1980 traf dies nur auf rund 7\% der Frauen und 9\% der Männer zu (Statistik Austria 2010: 84, 87). 
Die angesprochene Tendenz zu einer stärkeren eigenständigen wirtschaftlichen Absicherung von Frauen ist vor diesem Hintergrund ebenfalls als „Versicherung“, nun jedoch für unvorhersehbare Brüche im Zusammenleben mit Partner und Kindern, interpretierbar.

\subsection{Fertilitätskrise und „Überalterung “}

Mit den aktuellen Geburtenraten (und ohne Berücksichtigung der Zuwanderung) ist in vielen westlichen Industrieländern die Reproduktion nicht mehr gewährleistet. Sinkende und geringe Geburtenraten ${ }^{10}$ reduzieren langfristig das Arbeitskräfteangebot und schwächen damit die wirtschaftliche Produktivität und das langfristige Wachstumspotential. ${ }^{11}$ In Verbindung mit der steigenden Lebenswartung führen geringe Geburtenraten zu einer Umkehrung der Alterspyramide und der beklagten „Überalterung“ der Gesellschaft. Dadurch wird vielfach auch die langfristige Finanzierbarkeit sozialer Sicherungssysteme als gefährdet angesehen.

\subsection{Bildungskrise}

Internationale Vergleichsstudien wie die PISA-Studie stellen für Deutschland und Österreich eine eher unterdurchschnittliche Leistungsfähigkeit der SchülerInnen bezüglich der abgefragten Kompetenzen fest. Viele SchulabgängerInnen können einfachste Lese-, Schreibbzw. Rechenaufgaben nicht sinnerfassend und problemorientiert aufnehmen und auch nicht eigenständig lösen. Sekundärer Analphabetismus und massive Bildungsdefizite werden auch bei der zweiten und dritten MigrantInnengeneration diagnostiziert. ${ }^{12}$ Die immer wieder festgestellte „Überforderung“ von Familien dürfte zum Teil auch mit diesbezüglichen (zeitlichen und fachlichen) Ansprüchen der Schule an die Eltern zu tun haben. Zudem weisen Österreich und Deutschland im Vergleich zu anderen OECD-Ländern ein hohes Ausmaß an sozialer Vererbung des Bildungserfolgs auf. ${ }^{13}$

\subsection{Noch immer unzureichende Geschlechtergleichstellung}

Die Herstellung von Geschlechtergerechtigkeit ist auf formal-rechtlicher Ebene zwar schon weitgehend umgesetzt und wird inzwischen auch als gesellschaftspolitisches Ziel weitgehend akzeptiert. Letzteres trifft - zumindest auf der rhetorischen Ebene - sogar für Gruppierungen zu, die dieses Ziel weltanschaulich gesehen nicht voll teilen, wie später an einem Beispiel gezeigt wird.

Trotz grundsätzlicher Akzeptanz und Verankerung dieses Ziels mangelt es jedoch auch im Bereich der Familienpolitik an einer einheitlichen Zieldefinition und damit einhergehend auch vielfach an einer entsprechenden praktischen Umsetzung. Ein Beispiel dafür ist die noch immer sehr geringe Beteiligung der Väter an der Kinderbetreuung und -erziehung.

Aus dieser Auflistung diverser Veränderungen von ökonomischen und gesellschaftlichen Rahmenbedingungen und daraus resultierenden Problemlagen wird ersichtlich, dass es grundsätzlich starke ökonomische und außerökonomische Triebkräfte für eine Anpassung der Familienpolitik an die neuen Gegebenheiten gibt. Für die Beurteilung, ob und inwieweit sich das in Veränderungen familienpolitischer Grundsatzpositionen niedergeschlagen hat, werden im nächsten Abschnitt entsprechende Kriterien festgelegt.

${ }^{10}$ Die Fertilitätsrate lag in Österreich im Jahr 2008 bei etwa 1,4 Kindern pro Frau, Anfang der 1960er Jahre lag sie noch bei rund 2,8 Kindern. Wo die ,richtige“ und damit anzustrebende Geburtenrate liegt, ist aus einer globalen und langfristigen Perspektive, die auch die weltweiten Wanderungsbewegungen sowie ökologische Probleme einschließt, nicht so einfach zu beantworten. Es gibt aber gute Gründe anzunehmen, dass sie in der Regel nicht mit einer aus nationalstaatlicher Sicht bestandssichernden Fertilitätsrate zusammenfallen dürfte.

11 Vgl. dazu und zum Folgenden insb. die Abschnitte 3. 1 bzw. 3.2 im Beitrag von Schratzenstaller in diesem Band mit entsprechenden empirischen Belegen.

12 Vgl. etwa Steinmayr (2009) zur Bildungssituation der zweiten MigratInnengeneration in Wien.

13 Vgl. dazu Wößmann (2009) und Jacob (2009) für Deutschland sowie den Abschnitt 3.5 im Beitrag von Schratzenstaller in diesem Band für Österreich und mit weiterer Literatur. 


\section{$3 \quad$ Kriterien eines Paradigmenwechsels}

Die dargestellten faktischen Veränderungen und laufenden Diskurse können zum einen Zielen der Familienpolitik zugeordnet werden, bei denen Gerechtigkeitsaspekte im Vordergrund stehen. Dazu zählt klarerweise die Gleichstellung der Geschlechter. Bezüglich der Kinder betrifft das Ziele wie Chancengleichheit im Bildungswesen und soziale Integration, die letztlich zur Stärkung des gesamtgesellschaftlichen Zusammenhalts führt. Zum anderen lassen sich damit ökonomisch begründete Ziele wie die Sicherung bzw. Steigerung gesamtwirtschaftlicher Produktivität und von wirtschaftlichem Wachstum sowie die Sicherung der Finanzierbarkeit von Sozialsystemen in Verbindung bringen. ${ }^{14}$ Teilziele, die diesen Zielbündeln zugeordnet werden können, werden im nächsten Abschnitt als Kriterien für die Beurteilung eines allfälligen Paradigmenwechsels in seinen verschiedenen Dimensionen bei familienpolitischen Positionen - und Maßnahmen im Beitrag von Kreimer in diesem Band - dienen. Vorerst erfolgt eine kurze Darstellung und Diskussion dieser Ziele, Teilziele und Zielindikatoren.

\subsection{Geschlechtergleichstellung}

Diesem Ziel können Teilziele wie gleiche Zugangschancen für Mütter zu Beschäftigung und Karriere bei gleichzeitiger eigenständiger, vom Partner unabhängiger Existenzsicherung zugeordnet werden. Im nächsten Abschnitt wird anhand der folgenden Fragen untersucht, ob in den grundsätzlichen familienpolitischen Positionen von Parteien und Interessenvertretungen in Österreich eine Umorientierung in Richtung folgender gleichstellungspolitisch begründeter Teilziele erkennbar ist:

- Werden für Mütter (im Vergleich zu kinderlosen Frauen und Männern bzw. Vätern) gleiche Teilhabechancen am Arbeitsmarkt - die sich unter anderem in einer steigenden Erwerbsquote äußern - angestrebt? Wird dabei auch eine eigenständige finanzielle $A b$ sicherung der Frauen in der Berufs- und perspektivisch auch der Pensionsphase ${ }^{15}$ verfolgt?

- Werden spiegelbildlich zum vorhergehenden Argument für die Väter gleiche Teilhabechancen in der Familie - also in der Kinderbetreuung und -erziehung - angestrebt?

Falls die Analyse der Entwicklung familienpolitischer Ziele bzw. der zu ihrer Erreichung vorgeschlagenen Maßnahmen eine eindeutige Tendenz in diese Richtung zeigt, wäre das modernisierte traditionelle Paradigma mit seiner primären Zuständigkeit der Mütter für die Familienarbeit in Richtung einer geteilten Zuständigkeit von Männern und Frauen samt eigenständiger Absicherung durchbrochen. In diesem Fall wird eine mehr oder weniger starke Abkehr von diesem Paradigma diagnostiziert.

\subsection{Steigerung von Produktivität und Wachstum sowie Sicherung der Sozialsysteme}

Zur Erreichung der ökonomischen Ziele der Erhaltung bzw. Steigerung der gesamtwirtschaftlichen Produktivität und von Wirtschaftswachstum sowie der Sicherstellung einer dauerhaften Finanzierung von sozialen Sicherungssystemen werden unter anderem vorgeschlagen:

- Maßnahmen, die auf eine möglichst gute Entwicklung bzw. hohe Ausschöpfung des Humanvermögens von Kindern bzw. Frauen und somit einer Steigerung der Frauenerwerbsquote abzielen;

- eine pro-natalistische Familienpolitik der Steigerung der Geburtenrate; ${ }^{16}$

- Zuwanderung und Integration von MigrantInnen.

${ }^{14}$ Vgl. dazu die Darstellung familienpolitischer und anderer damit verknüpfter Ziele im Abschnitt 2 des Beitrags von Schratzenstaller in diesem Band samt Diskussion der Zielbeziehungen.

15 Die eigenständige finanzielle Absicherung von Frauen in der Pension wird in diesem Beitrag nicht explizit untersucht. Allerdings ist in erwerbszentrierten Sozialversicherungssystemen die Verbindung von aktiver Erwerbstätigkeit und Pension in hohem Ausmaß gegeben, sodass sich Berufsunterbrechungen, Teilzeitphasen und geringe Einkommen jedenfalls auf die Pensionsansprüche auswirken.

${ }^{16}$ Es ist offensichtlich, dass die beiden letztgenannten Zielsetzungen nicht unter allen Umständen und so ohne weiteres konfliktfrei miteinander vereinbar sind. Ein Beispiel dafür, dass auch die folgenden, ökonomisch begründeten Teilziele untereinander, aber auch mit den anderen Teilzielen in Konflikt stehen können. 
Insbesondere der letztgenannte Vorschlag ist in Österreich noch mit massivem gesellschaftspolitischem Widerstand konfrontiert. Er weist starke familienpolitische Komponenten auf, führt aber auch zu zusätzlichen Herausforderungen in den Bereichen des Bildungswesens, der Integration und der sozialen Kohäsion, die beim folgenden bildungspolitischen Zielbündel detaillierter dargestellt werden.

Neben der Zuwanderung junger MigrantInnen kann auch eine verbesserte Vereinbarkeit von Erwerbsarbeit und Familie zur Erhöhung der Fertilitätsraten beitragen. Es ist allerdings umstritten, welche Maßnahmen und Rahmenbedingungen dafür zweckmäßig sind: Empirisch gesehen sind die Geburtenraten in Ländern mit höherer Frauenerwerbsquote tendenziell höher als in Ländern mit geringerer Frauenbeschäftigung. ${ }^{17}$ Demgemäß fördert unter anderem ein breit ausgebautes System bedarfsangepasster, flexibler außerfamiliärer Kinderbetreuungseinrichtungen, welches die Vereinbarkeit von Beruf und Familie auch in qualifizierten Berufen und auf hohem Beschäftigungsniveau mit relativ kurzen Ausstiegszeiten der Frauen ermöglicht, die Fertilität. Im Rahmen des modernisierten traditionellen Paradigmas wird dagegen weiterhin primär die innerfamiliäre Kinderbetreuung mit einem eher längeren, teilweisen oder vollständigen Ausstieg aus dem Berufsleben favorisiert. Der außerfamiliären Betreuung wird eine nur subsidiäre Rolle zugewiesen.

Aus ökonomischer Sicht sollte jedoch die mit der letztgenannten familienbezogenen Variante verbundene tendenzielle Entwertung des Humanvermögens von Müttern möglichst vermieden werden. Argumentationslinien, die auf die bestmögliche Realisierung der individuellen bzw. gesamtwirtschaftlichen Bildungsrenditen und der gesamtwirtschaftlichen Wachstumsmöglichkeiten abzielen, ${ }^{18}$ werden in der Folge vom gleichstellungspolitischen Diskurs unterschieden. Letzterer kann zwar zu analogen Ergebnissen bezüglich der Erwerbstätigkeit von Frauen führen, doch er bezieht seine argumentative Kraft nicht aus humankapitaltheoretischen, sondern aus gerechtigkeitstheoretischen Überlegungen.

Schließlich ist es zwar weitgehend unbestritten, dass für Länder wie Österreich eine qualitativ hochwertige (Aus-)Bildung der Kinder und Jugendlichen eine notwendige Voraussetzung zur Erreichung der obigen ökonomischen Ziele ist. Offensichtlich sind aber viele Familien, Kindergärten und Schulen in Österreich, aber auch in Deutschland, nicht in der Lage, den von Bildungsmängeln betroffenen Kindern geeignete inhaltliche und zeitliche Unterstützung zu geben, die ihnen das Erlernen der heutzutage unverzichtbaren primären Kulturtechniken ermöglicht, wie zuvor unter dem Schlagwort „Bildungskrise“ schon grob skizziert worden ist.

Bei diesem Teilziel, welches neben der Familien- vor allem auch die Bildungspolitik betrifft, ist zu untersuchen, ob in den Positionen der Parteien und Interessenvertretungen ein Wandel vom vorherrschenden Betreuungsparadigma hin zu einem Bildungsparadigma zu beobachten ist. Beim Erstgenannten steht die schon besprochene Ermöglichung von Vereinbarkeit von Erwerbsarbeit und Familie im Vordergrund. Dagegen zielt das Bildungsparadigma primär auf die Entwicklung des Humanvermögens des Kindes. Im Unterschied zum modernisierten traditionellen Paradigma wird davon ausgegangen, dass nicht zwangsläufig die Eltern (die Mutter) in allen Aspekten der Kindeserziehung die höchste Kompetenz besitzen und eine qualitativ hochwertige außerfamiliäre Betreuung quasi nur eine Notlösung ist. Gestützt durch empirische Untersuchungen wird vielmehr davon ausgegangen, dass außerfamiliäre Institutionen der Kinderbetreuung für die Entwicklung des Kindes wichtige Kompetenzen einbringen können, die im Rahmen der Familie in dieser Form nicht erbracht werden können. Zudem führen qualitativ hochwertige frühkindliche außerfamiliäre Bildungsanstrengungen aufgrund selbstverstärkender Effekte zu höheren Bildungsrenditen als Bildungsinvestitionen zu einem späteren Lebenszeitpunkt (Bock-Schappelwein et al. 2009: 25f., 59). Die ökonomischen Argumente der Vorteile von Spezialisierung und Arbeitsteilung werden in einem solchen neuen Paradigma auf den Reproduktionsbereich ausgedehnt, allerdings im Unterschied zum (modernisierten) traditionellen Paradigma - über den Kreis der Familie hinaus.

Maßnahmen wie ein Ausbau der außerfamiliären Kinderbetreuung und -erziehung auch schon in der frühkindlichen Phase, ganztägige Betreuungsangebote bis hin zur

17 Vgl. dazu die von Schratzenstaller im Abschnitt 2.4 angegebene Literatur.

$18 \mathrm{Zu}$ empirisch gestützten positiven Zusammenhängen zwischen der Erwerbsbeteiligung von Frauen bzw. der Humanvermögensentwicklung von Frauen und Kindern mit der wirtschaftlichen Produktivität vgl. die Abschnitte 3.4 und 3.5 im Beitrag von Schratzenstaller in diesem Band. 
Ganztagsschule, die Verbesserung der Qualität der Ausbildung der Betreuungspersonen sowie der Ausstattung der außerfamiliären Betreuungseinrichtungen mit qualifiziertem und differenziert ausgebildetem Personal, sind Indikatoren für einen solchen Wechsel zu einem Bildungsparadigma. Das gilt insbesondere dann, wenn die Maßnahmen verpflichtend sind.

Eine diesbezügliche Einschränkung der Autonomie der Familien, die den Kindern durch die Auferlegung entsprechender Verpflichtungen eine bestmögliche Entfaltung ihrer individuellen Potentiale außerhalb der Familie ermöglichen soll, stößt in Österreich derzeit allerdings noch auf breite Akzeptanzprobleme. In diesem Zusammenhang werden eine „Entmachtung“ der Familien und der Mütter und eine einseitig indoktrinierende „Verstaatlichung der Bildung" als Gefahr in den Raum gestellt. ${ }^{19}$ Solche berücksichtigungswürdigen und diskutablen Positionen vernachlässigen allerdings vielfach, dass eine Beibehaltung des familienbezogenen Leitbildes auf Kosten der bestmöglichen Entwicklung des Humanvermögens vieler Kinder und Jugendlichen geht und damit auch zu gesellschaftlichen Kosten führt.

Vor diesem Hintergrund ergeben sich folgende Fragestellungen zur Beurteilung, ob derartige, primär ökonomisch begründete Paradigmenwechsel vorliegen:

- Werden die Teilziele einer quantitativ besseren Nutzung des Arbeitskräftepotentials von Müttern bzw. einer qualitativ verbesserten Nutzung des Erwerbspotentials vor allem besser ausgebildeter Frauen und Mütter sowie dafür geeignete Maßnahmen angestrebt und explizit - unabhängig von gleichstellungspolitischen Zielen - mit den oben angeführten ökonomischen Argumenten begründet?

- Auf welchem Weg soll angesichts des demographischen Wandels und der daraus resultierenden ökonomischen Probleme eine Erhöhung der Geburtenrate erreicht werden? Geschieht dies vor einem Hintergrund, der eine - ökonomisch begründete - Erhöhung der Erwerbsbeteiligung von Müttern als vorteilhaft bzw. notwendig für die Zielerreichung ansieht?

- Zielt die Bildungs- und Familienpolitik unter Berufung auf die genannten ökonomischen Ziele sowie unter Verwendung humankapitaltheoretischer Argumente auf eine qualitativ hochwertige außerfamiliäre Betreuung und Ausbildung der Kinder und Jugendlichen? Wichtige Beurteilungskriterien dabei sind, ob dies auch für die frühkindliche Lebensphase vorgesehen ist und/oder ob die Angebote ganztägig bzw. verpflichtend sind.

Falls die Analyse der familienpolitischen Positionen und daraus abgeleiteter Maßnahmen eine eindeutige Tendenzen in diese Richtungen zeigt und zur Begründung jeweils auch Ziele wie Sicherung von Produktivität, Wachstum bzw. der Finanzierbarkeit der Sozialsysteme herangezogen werden, wird ein ökonomisch begründeter Paradigmenwechsel diagnostiziert. Auch in diesen Fällen würde das eine Abkehr vom modernisierten traditionellen Paradigma bedeuten.

\subsection{Chancengleichheit, soziale Integration und gesellschaftlicher Zusammenhalt}

Neben den eben besprochenen ökonomischen Problemen resultiert aus der „Bildungskrise“ auch ein Gerechtigkeitsproblem. Für Kinder aus bildungsfernen Schichten ist keine Chancengleichheit im und durch das Bildungssystem gegeben. Familien- und bildungspolitische Maßnahmen, die mehr Chancengleichheit für Kinder insbesondere aus bildungsfernen Schichten herstellen, leisten auch einen Beitrag zu späterer Armutsvermeidung: Verbesserte Bildung insbesondere bereits im frühkindlichen Alter verringert die soziale Vererbung von Bildungs- und damit späteren Einkommenschancen (Bock-Schappelwein et al. 2009: 38f.). Sie verändert langfristig die primäre Einkommensverteilung zugunsten dieser bildungsfernen Familien.

Zudem könnten entsprechende Maßnahmen zu einer verbesserten sozialen Integration von Kindern, insbesondere auch - aber nicht nur - aus MigrantInnenfamilien, und zu einer Stärkung der sozialen Kohäsion führen. Das betrifft die Vermittlung von Sprachkompetenzen ebenso wie sonstige integrationsfördernde Maßnahmen. Bereits das Miteinander von Kindern mit unterschiedlichen Begabungen und aus unterschiedlichen sozialen und kulturellen

19 Das kann sowohl auf die Verfolgung bestimmter familienpolitischer Leitbilder aber auch auf schichtspezifische, den eigenen Status bewahrende Interessen zurückgeführt werden und spiegelt sich in den familienpolitischen Positionen mancher Parteien wider, wie in der Folge noch gezeigt wird. 
Milieus in Kindergarten und Schule kann den sozialen Zusammenhalt stärken. ${ }^{20}$ Demgemäß ist in diesem Zusammenhang zu fragen:

- Wird eine durchgehende Überwindung des in Österreich noch immer sehr stark ausgeprägten schichtspezifisch selektiven Bildungszugangs als Ziel formuliert? Werden dafür geeignete Maßnahmen wie ein auch schon frühkindlicher, teils verpflichtender, ganztägiger und gemeinsamer Besuch von qualitativ hochwertigen Betreuungseinrichtungen und Schulen bzw. ihre Ausstattung mit quantitativ ausreichendem und qualitativ hochwertigem Betreuungspersonal vorgeschlagen?

- Betreffen diese Ziele und Maßnahmen auch den Abbau von Sprachdefiziten bei Kindern aus MigrantInnenfamilien und bildungsfernen Schichten sowie die Erhöhung der sozialen Kompetenz und der sozialen Integration der Kinder? Werden sozial durchmischte Betreuungseinrichtungen und Schulen als Instrument sozialer Integration und zur Stärkung des gesellschaftlichen Zusammenhalts gesehen?

Entsprechende Veränderungen in den familienpolitischen Positionen würden eine Abkehr vom (modernisierten) traditionellen Paradigma bedeuten, welches den primären Hort von Erziehung und Ausbildung weiterhin in der Familie sieht, was einer Erhaltung eines schichtspezifischen Zugangs zu Bildungserfolgen Vorschub leistet und zudem nicht integrationsförderlich ist.

Die Verbesserung der Vereinbarkeit von Erwerbsarbeit und Familie wurde bisher nur am Rande erwähnt, obwohl sie programmatisch immer wieder als wichtiges Ziel der Familienpolitik genannt wird. Doch unseres Erachtens ist dies nur ein Zwischenziel, welches den übergeordneten, oben spezifizierten Zielen auf direktem oder indirektem Weg förderlich sein kann, aber nicht muss. Deshalb wird die Vereinbarkeit in den folgenden Qualifizierungen der familienpolitischen Positionen entscheidungsrelevanter politischer Parteien und Interessensverbände nicht als eigenständiges Ziel, sondern nur jeweils gemeinsam mit den übergeordneten Teilzielen analysiert.

20 Ein maßgeblicher Vertreter dieser Position ist u. a. Bernd Schilcher (Mitarbeiter am 1972 verabschiedeten Salzburger Programm der Österreichischen Volkspartei, langjähriger Amtsführender Präsident des Landesschulrates für die Steiermark, Vorsitzender einer Expertenkommission zur Schulreform des zuständigen Bundesministeriums etc.). Er hat sie in seinem Referat beim Symposium „Paradigmenwechsel in der Familienpolitik?“ des Schumpeter Centers an der Universität Graz am 15. und 16. April 2010 überzeugend vertreten. 


\section{Entwicklung familienpolitischer Positionen in ausgewählten politischen Parteien und Interessenvertretungen}

Im Folgenden wird unterstellt, dass sich ein Paradigmenwechsel in den Positionen entscheidungsrelevanter Akteure zur Familienpolitik (und den damit verknüpften Bereichen der Bildungs- und Arbeitsmarktpolitik) finden lassen müsste. ${ }^{21}$ Es wird untersucht und in der folgenden Tabelle 1 zusammenfassend dargestellt, inwieweit die obigen Zielsetzungen, Begründungen für neue Zielsetzungen und zur Erreichung dieser Ziele vorgeschlagenen Maßnahmen in den Positionen ausgewählter politischer Parteien und Interessenvertretungen in Österreich ihren Niederschlag gefunden haben. $\mathrm{Zu}$ diesem Zweck werden diverse Partei- und Wahlprogramme bzw. Zukunfts- und Grundsatzpapiere der vergangenen Jahrzehnte der Österreichischen Volkspartei (ÖVP), der Freiheitlichen Partei Österreichs (FPÖ), der Sozialdemokratischen Partei Österreichs (SPÖ) sowie der Industriellenvereinigung (IV) und der Sozialpartner analysiert. ${ }^{22}$

Zwecks Eingrenzung des breiten Analysefeldes beschränken wir uns auf zwei wesentliche Teilbereiche der Familien- und Bildungspolitik: Zum einen auf die institutionalisierte, außerhäusliche (außerfamiliäre) Kinderbetreuung und deren Ausgestaltung und zum anderen auf den Bereich der Elternkarenz und der (teils damit verbundenen) finanziellen Unterstützung der Familien wie dem Kinderbetreuungsgeld (KBG). ${ }^{23}$

Es sei vorausgeschickt, dass angesichts der vielfältigen Wechselwirkungen und Beziehungen zwischen den einzelnen Zielen und Maßnahmen und vor allem angesichts der nicht widerspruchsfreien und eindeutigen Darstellung der Positionen bei den analysierten familienpolitischen Institutionen nur grobe grundsätzliche Einschätzungen ihrer Entwicklung vorgenommen werden können, die da und dort erforderliche Differenzierungen vernachlässigen. Das Ziel dieser Arbeit geht aber auch über die Erlangung solcher Tendenzaussagen, die dann mit den ebenfalls nur grob charakterisierbaren realisierten Maßnahmen kontrastiert werden können, nicht hinaus. Detaillierte Analysen müssen anderen Arbeiten (insbesondere auch politikwissenschaftlicher Natur) vorbehalten bleiben. ${ }^{24}$

21 Es ist in der Regel nicht zu erwarten, dass Veränderungen politischer Grundsatzpositionen sehr zeitnah zu real auftretenden Problemen erfolgen, sondern vielmehr, dass sie einen gewissen zeitlichen Vorlauf benötigen. Da sich die oben dargestellten Probleme jedoch schon seit längerem abzeichnen, ist die Erwartung nicht unbegründet, dass sich dies schon in den Grundsatzpositionen niedergeschlagen haben könnte.

Vgl. dazu das Modell von Hall (1993) zum politischen und gesellschaftlichen Lernen. Demnach führen gesellschaftliche Krisen, die mit den vorherrschenden Paradigmen nicht mehr erklärt und mit den bisher verwendeten Instrumenten nicht mehr bewältigt werden können, zu stufenweisen, kumulativen Lernprozessen in Gesellschaft und Politik. Das gipfelt letztlich in der politischen Durchsetzung eines neuen Paradigmas mit neuen Zielen und Instrumenten und deren administrativer Verankerung. Dazu und zum Konzept des Paradigmenwechsels im Allgemeinen vgl. den abschließenden Abschnitt im Beitrag von Kreimer in diesem Band.

22 Regierungsprogramme werden in diese Analyse nicht einbezogen, da die analysierten Positionen möglichst unbeeinflusst von aktuellen tagespolitischen Notwendigkeiten und erforderlichen politischen Kompromissen bzw. Abtauschgeschäften im Zuge von Koalitionen bzw. Regierungsverhandlungen sein sollen.

23 Vgl. dazu den Beitrag von Leitner in diesem Band, der diese Auswahl stützt. Leitner sieht u. a. einkommensabhängige und existenzsichernde Transferleistungen für Zeiten der Kinderbetreuung sowie quantitativ umfassende, flexible und qualitativ hochstehende Kinderbetreuung als zentrale Bausteine einer guten Familienpolitik.

24 Eine teils umfassendere, aber vorläufige und aktuelle Entwicklungen noch nicht beinhaltende Darstellung familienpolitischer Positionen der hier analysierten Institutionen findet sich in Dujmovits (2009). 
Tabelle 1: Entwicklung familienpolitischer Positionen in Österreich

\begin{tabular}{|c|c|c|c|c|c|}
\hline Zielebenen*) & $\begin{array}{c}\text { (1) } \\
\text { Gleichstellung }\end{array}$ & \multicolumn{3}{|c|}{$\stackrel{(2)}{\text { Ökonomische Ziele }}$} & $\begin{array}{c}\text { (3) } \\
\text { Chancen- } \\
\text { gleichheit }\end{array}$ \\
\hline $\begin{array}{l}\text { Teilziele bzw. } \\
\text { Zielindikato- } \\
\text { ren }\end{array}$ & $\begin{array}{l}\text { eigenständige } \\
\text { und gleiche } \\
\text { Teilhabechan- } \\
\text { cen in Beruf und } \\
\text { Familie für Frau } \\
\text { \& Mann }\end{array}$ & $\begin{array}{l}\text { umfassende } \\
\text { Nutzung } \\
\text { Humanver- } \\
\text { mögen } \\
\text { Frauen \& } \\
\text { Mütter }\end{array}$ & $\begin{array}{c}\text { Steigerung } \\
\text { Geburtenrate } \\
\text { durch Verein- } \\
\text { barkeit auf ho- } \\
\text { hem Niveau }\end{array}$ & $\begin{array}{r}\mathrm{I} \\
\mathrm{Hu} \\
\text { gen } \\
\mathrm{Vc}\end{array}$ & $\begin{array}{l}\text { jrderung } \\
\text { lanvermö- } \\
\text { ntwicklung } \\
\text { Kindern }\end{array}$ \\
\hline \multicolumn{6}{|c|}{ Veränderungen $\left.{ }^{* *}\right)$ in den Positionen der ... } \\
\hline ÖVP & $(+)$ & $=$ & $=$ & $(+)$ & $(+)$ \\
\hline FPÖ & - & - & - & $=$ & $=$ \\
\hline SPÖ & ++ & $=$ & $=$ & $(+)$ & ++ \\
\hline $\begin{array}{c}\text { IV \& Sozial- } \\
\text { partner }\end{array}$ & ++ & ++ & $=$ & ++ & ++ \\
\hline
\end{tabular}

Quelle: eigene Zusammenstellung

*) Zielebenen:

(1) Geschlechtergleichstellung

(2) Sicherung von Produktivität, Wirtschaftswachstum sowie der Finanzierbarkeit der Sozialsysteme

(3) Chancengleichheit in/ durch Bildung; soziale Integration und Kohäsion.

**) Ausmaß und Richtung der Veränderung: modernisiertes male-breadwinner-Modell als Referenzpunkt

++ deutliche Hinwendung zu einem neuen Paradigma

$+\quad$ Hinwendung zu einem neuen Paradigma

(+) sehr schwache Hinwendung zu einem neuen Paradigma

$=$ unverändert

- $\quad$ Rückwendung zum traditionellen Paradigma, mit starker Betonung innerfamiliärer Kindererziehung.

\section{1 ÖVP: Wahlfreiheit versus grundsätzlich subsidiärem Charakter außerhäuslicher Kinderbetreuung}

Angesichts ihrer seit 1987 durchgehenden direkten bzw. indirekten politischen Verantwortung für die Familienpolitik sind die diesbezüglichen politischen Positionen der ÖVP von besonderem Interesse. ${ }^{25}$

Grundsätzlich wird in den analysierten Positionspapieren der ÖVP ${ }^{26}$ durchgehend die Schaffung bzw. Erhaltung von Wahlfreiheit als wesentliches Ziel bei der Ausgestaltung der Vereinbarkeit von Erwerbsarbeit und Familie, der Kinderbetreuung und der Schule hervorgehoben. ${ }^{27}$ Eine kritische Einordnung dieser Position erfolgt in der zusammenfassenden Würdigung der einzelnen Standpunkte im letzten Abschnitt dieses Beitrags.

Das Ziel der Geschlechtergleichstellung wird auf allgemeiner Ebene mehrfach explizit angesprochen. An Maßnahmen, die dazu führen, werden im Parteiprogramm bedarfsbezogene, die Familie subsidiär unterstützende und die Vereinbarkeit von Erwerbsarbeit und Familie erleichternde Angebote an vorschulischer Kinderbetreuung genannt (ÖVP 1998: 18,

${ }^{25}$ Die ÖVP war zwischen 1987 und 2000 für die Familienpolitik politisch zuständig und ist es ab dem Jahr 2007 wiederum. Zwischen 2000 und 2007 war das Familienministerium in der Hand der FPÖ, später dem Bündnis Zukunft Österreich (BZÖ), jeweils in einer Koalitionsregierung mit der ÖVP.

26 Das sind: i) Das Parteiprogramm aus dem Jahr 1995 (ÖVP 1998). ii) Das im Rahmen des ÖVP-Perspektivenprozesses 2010 erarbeitete Ergebnispapier der Impulsgruppe Familie und Kinder (Mikl-Leitner 2007) sowie das unter anderem darauf bauende Perspektivenpapier (ÖVP 2007). Letzteres soll zwar die Grundlage für ein neues Parteiprogramm bilden, werde aber „nur einen Teil dazu beitragen“ und auch „nicht die ,Bibel' sein“ (Die Presse 2009). Es kann daher als politisch ziemlich unverbindlich eingestuft werden. Diese Einschätzung findet ihre Bestätigung durch die teils unterschiedlichen Positionen in Mikl-Leitner (2007) und ÖVP (2007) sowie im Vergleich mit Positionspapieren jüngeren Datums. iii) Das Wahl- (ÖVP 2008) und Frauenprogramm (ÖVP-Frauen 2008) für die Nationalratswahl 2008, in denen zum Teil wieder stärker traditionelle Positionen vertreten werden. iv) Das ÖVP-Bildungskonzept (ÖVP 2011).

27 Vgl. dazu (ÖVP 1998: 17; 2008: 7, 11). Laut Perspektivengruppe soll die Wahlfreiheit der Eltern bei der Kinderbetreuung (Mikl-Leitner 2007: 8; ÖVP 2007: 10f.) und in der Schule (ÖVP 2007: 22) erhöht werden; letztere soll laut dem jüngsten Bildungskonzept „die Wahlfreiheit in den Mittelpunkt stellen“(ÖVP 2011: 2). 
24). Die Impulsgruppe Familie ging einen Schritt weiter und schlug eine Flexibilisierung der Öffnungszeiten und eine Reduktion der Schließtage von (öffentlichen) Kinderbetreuungseinrichtungen vor (Mikl-Leitner 2007: 8). Im Perspektivenpapier folgte ein weiterer großer Schritt: Es wurde eine bedarfsgerechte Betreuung (Tagesmütter, Kinderkrippe) auch für unter 3-Jährige sowie ein kostenloser Kindergarten am Vormittag ab dem 4. Lebensjahr gefordert (ÖVP 2007: 11, 19). Im Programm für die Nationalratswahl 2008 finden sich jedoch insbesondere die Vorschläge für die unter 3-Jährigen nicht mehr: Es wird nun ein kostenloser (und erstmals verpflichtender!) Kindergarten ab dem 5. Lebensjahr sowie erstmals ein bedarfsorientiertes, flächendeckendes Angebot an Kinderbetreuung auch für den Nachmittag gefordert (ÖVP 2008: 7). Weiters soll es eine „Betreuungsgarantie“ für Kinder in einer Einrichtung nach Wahl der Eltern geben (ÖVP 2008: 11). Laut dem jüngsten ÖVP-Bildungskonzept sollen in den Schulen „bedarfsorientiert flächendeckende, ganztägige Betreuungsangebote“ geschaffen werden. Für alle 3- bis 6-jährigen Kinder soll es ein „bedarfsorientiertes flächendeckendes Angebot" (ÖVP 2011: 2f.) geben. ${ }^{28}$

In Österreich ist das Angebot an ganztägigen Kinderbetreuungseinrichtungen insbesondere für Kleinkinder unter drei Jahren, aber auch für 6- bis 14-jährige Schulkinder in der Nachmittagsbetreuung generell zu gering. Zudem gilt für viele Einrichtungen, dass sie in Bezug auf tägliche Öffnungszeiten und ferienbedingte Schließzeiten auf die beruflichen Anforderungen der Eltern bzw. deren Urlaubsrechte zu wenig Rücksicht nehmen (Fuchs 2006). ${ }^{29}$ Angesichts dieser Mängel müsste ein rascher, flächendeckender Ausbau entsprechender Einrichtungen und die Bereitstellung der dafür erforderlichen Mittel gefordert werden, doch das findet sich in den aktuellsten Grundsatzpapieren der ÖVP nicht. Die jüngsten Vorschläge fallen hinter jene im Perspektivenpapier bzw. im Wahlprogramm zurück. Das flächendeckende Betreuungsangebot soll nun offensichtlich für unter 3-Jährige nicht gelten bzw. wird auch kein ganztägiges Angebot in Kindergärten gefordert. Unter anderem wird daraus deutlich, dass in der ÖVP bezüglich der Kinderbetreuung noch keine eindeutige und dauerhafte Tendenz erkennbar ist, die u. a. eine stärkere Gleichstellung von Mann und Frau stützt.

Zur Erleichterung der Vereinbarkeit von Beruf und Familie wird weiters der Ausbau qualifizierter Teilzeitarbeit und von flexiblen Arbeitszeitmodellen propagiert (ÖVP 1998: 14; Mikl-Leitner 2007: 10; ÖVP 2007: 14). In Mikl-Leitner (2007: 12) wird eine gänzliche Abschaffung der Zuverdienstgrenze beim Kinderbetreuungsgeld (KBG) vorgeschlagen. Im Perspektivenpapier und in den folgenden Wahlprogrammen findet sich diese Forderung nicht mehr. In ÖVP (2008: 11) wird aber ein einkommensabhängiges KBG für ein Jahr vorgeschlagen. ${ }^{30} \mathrm{Im}$ Unterschied zu den langen Varianten des $\mathrm{KBG}^{31}$ in nicht existenzsichernder Höhe - die zwecks Aufrechterhaltung von Wahlfreiheit nicht in Frage gestellt werden - kann eine solche einkommensabhängige Variante grundsätzlich die Arbeitsmarktbeteiligung von Müttern (und auch die Väterkarenz) fördern, ${ }^{32}$ falls es nach diesem Jahr ausreichende Betreuungseinrichtungen für Kleinkinder gibt. Da dies gerade bis zum Alter von drei Jahren praktisch gesehen nur sehr eingeschränkt der Fall ist und sich auch in den jüngsten Forderungen der ÖVP nichts in diese Richtung findet, ist auch diese Positionsänderung kein klares Signal in Richtung eines gleichstellungspolitischen Paradigmenwechsels. Zudem wird mehrfach die Einführung eines Familiensplittings in der Einkommensbesteuerung gefordert (ÖVP 2007: 17; 2008: 10f.), woraus sich tendenziell auch negative Anreize für die Erwerbstätigkeit typischerweise der Frau als Zweitverdienerin ergeben. ${ }^{33}$

\footnotetext{
${ }^{28}$ Weiters sollen Betriebskindergärten steuerlich gefördert werden (ÖVP 2008: 7). Forderungen nach einer steuerlichen Begünstigung familienfreundlicher Unternehmen finden sich auch in (Mikl-Leitner 2007: 11), dagegen wird im Perspektivenpapier für die Schaffung einer familienfreundlichen Arbeitswelt nur die „Verantwortung von Wirtschaft und Unternehmen“(ÖVP 2007: 11) bemüht.

${ }^{29}$ Vgl. dazu auch die Detailauswertung für Österreich im Beitrag von Kreimer in diesem Band.

${ }^{30}$ Diese Forderung wurde zwischenzeitlich umgesetzt. Vgl. dazu den Beitrag von Kreimer in diesem Band.

${ }^{31}$ In diesen wird das KBG für 30 (bzw. 20) Monate ausbezahlt, falls es nur von einem Elternteil in Anspruch genommen wird. Zu weiteren Details vgl. den Beitrag von Kreimer in diesem Band.

32 Analoges gilt insbesondere für besser qualifizierte Frauen für die steuerliche Absetzbarkeit von Kinderbetreuungskosten, die u. a. in ÖVP (2007: 17; 2008: 11) und Mikl-Leitner (2007: 8) vorgeschlagen wird und zwischenzeitlich ebenfalls verwirklicht ist.

${ }^{33}$ Um solche negativen Arbeitsanreize zu vermeiden, sprechen sich die ÖVP-Frauen (2008, Punkt 3) dagegen für die Beibehaltung der Individualbesteuerung aus.
} 
Schließlich wird zur grundsätzlich gewünschten verstärkten Beteiligung der Väter in die Familienarbeit inklusive Väterkarenz ${ }^{34}$ vor allem auf die Überwindung alter Rollenbilder gesetzt (ÖVP 2007: 13f.). Beispielsweise werden verpflichtende „Papamonate“ - die von der SPÖ vorgeschlagen werden - nicht gefordert bzw. abgelehnt, was angesichts des hohen Stellenwerts von Wahlfreiheit nicht wirklich überraschend ist.

Die ÖVP verbleibt demnach bezüglich des Ziels der Geschlechtergleichstellung noch weitgehend im Rahmen des modernisierten traditionellen male-breadwinner-Modells. Das Ziel einer umfassenden Gleichstellung von Mann und Frau in Beruf und Familie samt eigenständiger Absicherung berufstätiger Mütter wird zwar mehrfach artikuliert, die vorgeschlagenen Maßnahmenbündel weisen jedoch nicht eindeutig in diese Richtung, sind nicht umfassend genug und bleiben vielfach auf halbem Weg stehen. Beispielsweise soll zwar die qualifizierte Teilzeit ausgebaut werden, ein umfassendes Recht auf Elternteilzeit wird dagegen nicht explizit gefordert. ${ }^{35}$ Insbesondere die Betreuung unter 3-Jähriger bzw. am Nachmittag soll weiterhin primär in der eigenen Familie, vor allem bei den Müttern, verbleiben. Beispielsweise meinte die ÖVP-Wissenschaftsministerin Karl im Zuge der Diskussion des neuen ÖVP-Bildungskonzeptes, dass sich die Eltern zukünftig in Bildungsfragen mehr einbringen und mit den Kindern verstärkt Hausübungen machen sollen (ORF 2011). Die zwar vorhandenen, aber stark widersprüchlichen Anzeichen für eine stärker gleichstellungsorientierte Politik können nicht als klarer Wechsel in Richtung gleicher Teilhabechancen interpretiert werden. Diese nur sehr schwache Hinwendung zu einem neuen Paradigma führt in der ersten Spalte der Tabelle 1 zu einem eingeschränkten Plus - , (+)“

Dieses Ergebnis entspricht auch der grundsätzlichen Positionierung der ÖVP (1998: 18) in ihrem Parteiprogramm, wonach außerfamiliäre Kinderbetreuung, wie alle anderen Unterstützungsleistungen des Staates für Familien auch, „grundsätzlich subsidiären Charakter“ habe und die innerfamiliäre Betreuung nur ergänzen und bereichern soll. ${ }^{36}$

Auch bezüglich des Teilziels einer verstärkten Nutzung des Humanvermögens der Frauen ist kein Paradigmenwechsel zu erkennen. Einige der beschriebenen jüngsten Forderungen der ÖVP begünstigen zwar insbesondere bei besser verdienenden (ausgebildeten) Müttern eine frühere Aufnahme der Erwerbstätigkeit nach der „Babypause“. Doch obwohl es sich aus sachlichen Gründen aufdrängen würde, werden humankapitaltheoretische Argumente samt Bezügen zu den angeführten gesamtwirtschaftlichen Zielen nicht ${ }^{37}$ bzw. nur beiläufig verwendet (ÖVP 2007: 13, 38ff.). Diese fast völlige Ausblendung ökonomischer Argumente wird als unveränderte Positionierung eingestuft und mit einem ,,=“ in der zweiten Spalte der Tabelle 1 gekennzeichnet.

In den analysierten Positionspapieren werden diverse steuerpolitische und andere Maßnahmen zur Unterstützung von Zwei-(Mehr-)Kindfamilien vorgeschlagen. Eine höhere Geburtenrate soll vor allem durch ein „Familiensplitting österreichischen Zuschnitts“ (ÖVP 2008: 10f.) erreicht werden. ${ }^{38}$ Das entspricht jedoch nicht einer Hinwendung zu einem - primär ökonomisch begründeten ${ }^{39}$ - Teilziel einer Steigerung der Geburtenrate durch Vereinbarkeit von Erwerbsarbeit und Familie auf hohem Beschäftigungsniveau vor allem der Mütter. Die dargestellten halbherzigen Bekenntnisse zu einem umfassenden, ganztägigen Ausbau der Kinderbetreuungseinrichtungen in Verbindung mit Forderungen, die eine lange Abwesenheit vom Arbeitsmarkt begünstigen, erschweren den Wiedereinstieg ins Berufsleben. Diese Anreize zu innerfamiliärer Kindererziehung durch die Mütter und für längere

34 Vgl. dazu das Perspektivenpapier (ÖVP 2007: 10, 13f.) und weniger deutlich in Mikl-Leitner (2007: 14). Auch die ÖVP-Frauen (2008) wollen Väterkarenz fördern. Zu weiteren diesbezüglichen Maßnahmen siehe den Beitrag von Kreimer in diesem Band.

$35 \mathrm{Zu}$ den diesbezüglich bereits verwirklichten Maßnahmen vgl. den Beitrag von Kreimer in diesem Band.

36 In ÖVP (1998: 18) heißt es dazu auch: „Kinder brauchen Schutz, Geborgenheit und Orientierung an Werten, die ihnen in erster Linie von den Müttern und Vätern vermittelt werden“, wobei „die Bildungsverantwortung der Eltern gestärkt“ (24) werden müsse. Im jüngsten Bildungskonzept wird diese Position mit etwas anderen Worten wieder zum Ausdruck gebracht: „Die Eltern sind für die Erziehung und Ausbildung der Kinder verantwortlich und werden von der Schule in der Begleitung und Förderung ihrer Kinder unterstützt“ (ÖVP 2011: 2; ähnlich 2007: 23).

37 Beispielsweise soll laut dem damaligen ÖVP-Parteichef Wilhelm Molterer und der Staatssekretärin Christine Marek mit dem einkommensabhängigen KBG eine „,bessere Vereinbarkeit von Familie und Beruf“ ermöglicht und damit eine Antwort auf ,veränderte Lebensläufe“ (Presse 2008) gegeben werden.

38 Mikl-Leitner (2007: 6) schlagen „Anreize zum zweiten Kind“ vor, um das Auseinanderklaffen von Kinderwunsch und dessen Realisierung zu vermindern. Laut ÖVP (2007: 11, 17) ist ein Familiensplitting, bei dem die Steuerersparnis mit der Kinderzahl steigt, die wichtigste Maßnahme zur Geburtensteigerung.

39 Kurze, eher beiläufige Hinweise auf die ökonomische Bedeutung einer Steigerung der Kinderzahl finden sich nur in den unverbindlichen Perspektivenpapieren (Mikl-Leitner 2007: 6; ÖVP 2007: 12f.). 
Abwesenheiten vom Arbeitsmarkt verstärken sich mit steigender Kinderzahl. Das „,=“ in der dritten Spalte der Tabelle 1 drückt aus, dass die Erhöhung der Erwerbsbeteiligung der Mütter für die ÖVP noch kein Instrument zur Erhöhung der Geburtenrate ist.

Das Teilziel einer Förderung der Humanvermögensentwicklung von Kindern durch eine qualifizierte außerhäusliche Betreuung gewinnt in der ÖVP an Bedeutung. Bildung muss demnach bereits im Kindergarten, der zu einem „Lerngarten“ entwickelt wird, beginnen, weil ,auch die beste Familie kann einem Kind nicht alles mitgeben, was es für ein erfolgreiches Leben braucht“"(ÖVP 2007: 17f.). Dafür werden auch explizit ökonomische (humankapitaltheoretische) Argumente vorgebracht (Mikl-Leitner 2007: 6; ÖVP 2007: 20f.; 2008: 6). Abweichend vom Parteiprogramm wurde von der ÖVP $(2008: 7,11)$ erstmals ein verpflichtendes, halbtägiges und kostenloses letztes Kindergartenjahr vor dem Schuleintritt vorgeschlagen. ${ }^{40}$ Laut ÖVP (2011: 3f.) soll es zukünftig unter bestimmten Bedingungen auch am Nachmittag eine verpflichtende Betreuung und Förderung geben: Im Kindergarten und der Volksschule bei Sprachdefiziten, für die 10- bis 14-Jährigen, wenn der erforderliche Lernerfolg nicht erbracht wird.

Eine grundlegende Neuorientierung bezüglich ökonomischer Begründung und der konkreten Ausgestaltung der daraus abgeleiteten Maßnahmen ist aus all dem aber nicht abzuleiten: Die ökonomischen Argumente werden nicht in ihren verschiedenen Facetten (Produktivität und Wachstum, Finanzierung der Sozialsysteme) erörtert und auch nicht konsequent zu Ende gedacht. Das wird u. a. bei den vorgeschlagenen Maßnahmen ersichtlich: Wie dargestellt, bleiben die jüngsten Forderungen für die unter 3-Jährigen hinter früheren Positionen deutlich zurück. Ganztägige Betreuungsformen bleiben die Ausnahme für bestimmte Sonderfälle, im Normalfall soll der Unterricht in den Volks- und Hauptschulen nur am Vormittag erfolgen. Trotz aller nunmehrigen Bekenntnisse zur Bedeutung der Kindergärten und einer Qualitätssicherung bei den KindergartenpädagogInnen u. a. durch Zertifizierung (Mikl-Leitner 2007: 8; ÖVP 2007: 11) wird beispielsweise deren Höherqualifizierung in Form einer tertiären Ausbildung nicht gefordert ${ }^{41}$ bzw. von ÖVP-nahen Organisationen auch massiv abgelehnt. ${ }^{42}$ Maßnahmen wie die Verkleinerung der Gruppengrößen bzw. eine Erhöhung des Betreuungspersonals pro Gruppe werden in den Positionspapieren nicht erwähnt bzw. bleiben sehr vage und unverbindlich.

Insgesamt gesehen ist daher nur ein kleiner Schritt in Richtung einer stärker ökonomisch begründeten Förderung der Humanvermögensentwicklung von Kindern feststellbar. Diese nur sehr schwache Hinwendung zu einem neuen Paradigma führt zu einem eingeschränkten Plus in der vierten Spalte der Tabelle 1 bei der ÖVP.

Im jüngsten Bildungskonzept (ÖVP 2011), aber auch im Perspektivenpapier (ÖVP 2007: 20ff.) werden die Schaffung von Chancengleichheit ${ }^{43}$ in der und durch Bildung sowie soziale Integration als Leitlinien für die Schulreform genannt. Die Erlangung von Sprachkompetenzen stellt demnach den ,zentralen Schlüssel“ (ÖVP 2011: 2) für gesellschaftliche Integration dar, wie sich auch in der verpflichtenden Sprachförderung im Fall von Sprachdefiziten zeigt. Die Differenzierung zwischen AHS-Oberstufe und Neuer Mittelschule soll aber erhalten bleiben, (ganztägige) gemeinsame Schulformen für die 10- bis zu 14-Jährigen sind weiterhin nicht vorgesehen.

Damit bleibt trotz dieser grundsätzlichen Bekenntnisse die nach Ansicht vieler BildungswissenschaftlerInnen zu frühe Differenzierung in der Bildungslaufbahn, die zu einem sozial selektiven Bildungssystem führt, erhalten. Die Beiträge einer verpflichtenden sozialen Durchmischung und eines gemeinsamen Lernens von SchülerInnen mit unterschiedlichen Begabungen und besonderen Bedürfnissen zur Leistungssteigerung und sozialen Integration werden offensichtlich gering geschätzt. Als Mittel zur Verbesserung der sozialen Integration wird einseitig auf die Erlangung von Sprachkenntnissen gesetzt.

40 Diese Forderung wurde inzwischen realisiert. Vgl. dazu den Beitrag von Kreimer in diesem Band. Laut ÖVP (2007: 19) sollte der freiwillige Kindergarten am Vormittag bereits ab dem 4. Lebensjahr kostenlos sein.

41 Nur im politisch unverbindlichen Perspektivenpapier wird kryptisch für eine „Ausbildung des Fachpersonals auch auf ,universitärer' Ebene“ (ÖVP 2007: 19) plädiert. Vgl. dazu gegensätzlich die klaren Vorschläge der Grünen (Musiol/Fellerer o. J.) für eine tertiäre Ausbildung von KindergartenpädagogInnen.

42 Der Österreichische Gemeindebund, eine Interessensvertretung der Gemeinden, lehnt eine tertiäre Ausbildung von KindergartenpädagogInnen unter anderem wegen der dadurch erhöhten Kosten vehement ab (Hink 2009: 19).

43 Laut dem Perspektivenpapier ist „Bildungsgerechtigkeit“ anzustreben: „Die soziale Herkunft eines Menschen darf nicht über seine Zukunft entscheiden“ (ÖVP 2007: 44). 
Zudem werden Kompetenz und Verantwortung für die Bildung der Kinder auch in den jüngsten analysierten Positionspapieren der ÖVP weiterhin primär den Eltern zugeschrieben. Nicht das qualifiziert begleitete gemeinsame Lernen und Üben von unterschiedlich begabten Kindern auch mit unterschiedlichem kulturellem Hintergrund soll ausgebaut werden, sondern die Eltern sollen zukünftig ,stärker als bisher“ (ÖVP 2007: 23) in schulische Belange einbezogen werden. Wie gezeigt, wird sogar erwartet, dass sie ihre Kinder verstärkt bei den Hausübungen unterstützen. Es wird demnach nicht als vorrangige gesellschaftliche Aufgabe gesehen, das diesbezüglich offensichtliche Versagen vieler - insbesondere bildungsferner Familien bzw. auch ihre „Überforderung“ durch (verpflichtende) staatliche Maßnahmen auszugleichen. Insgesamt lässt sich auch hier nur eine sehr schwache Neuorientierung in Richtung eines neuen Paradigmas erkennen, wofür das eingeschränkte Plus bei der ÖVP in der letzten Spalte der Tabelle 1 steht.

\subsection{FPÖ: Steigerung der Geburtenrate durch Familiensplitting}

Zwischen 2000 und 2007 lag die Familienpolitik in der Händen der FPÖ (später BZÖ). In diese Zeit der Koalitionsregierung mit der ÖVP fällt die Einführung des unabhängig vom vorhergehenden Erwerbsstatus gewährten Kinderbetreuungsgeldes (KBG) ab 1. 1. 2002, welches das erwerbsarbeitszentrierte Modell des Karenzgeldes ersetzte.

Die familienpolitischen Positionen der FPÖ ${ }^{44}$ weisen eine starke Präferenz für die Erziehung und Betreuung von Kindern im Familienverband durch einen Elternteil (vorzugsweise die Mutter) auf. Demnach hat die Erziehung in der Familie grundsätzlich „Vorrang vor der Erziehung durch staatliche Einrichtungen“ (FPÖ 2005: 30), wobei ,gerade in den ersten Lebensjahren eines Kindes die Nähe der Mutter besonders wichtig und prägend ist" (FPÖ 2009: 126). Private und staatliche außerfamiliäre Betreuungsinstitutionen sollen nur soweit erforderlich ergänzend tätig sein (FPÖ 2007: 81; 2009: 133). Zum Zwecke des Kindeswohls muss es für Kleinkinder bis zum Kindergartenalter möglich gemacht werden, dass zumindest ein Elternteil die Kinder selbst betreuen kann. Betreuung durch Tagesmütter/-väter ist der Betreuung in Kinderkrippen vorzuziehen und auszubauen (FPÖ 2007: 83, 87; 2009: 131, 143).

In gleichstellungspolitischer Hinsicht wird zwar die Wahlfreiheit der Eltern betont, doch die Erhöhung der Erwerbstätigkeit von Eltern mit Kleinkindern wird explizit als kein anzustrebendes Ziel definiert (FPÖ 2007: 83; 2009: 131). Auch die geforderten familienpolitischen Maßnahmen wie Besteuerung mittels Familiensplitting (FPÖ 2005: 22ff.; 2007: 84f.; 2009: 137f.), Ausdehnung des KBG (samt Abschaffung der Zuverdienstgrenze) sowie des Karenzschutzes auf drei Jahre (später in Form eines Kindererziehungsgeldes bis zur Schulpflicht), Ablehnung eines einkommensabhängigen KBG etc. setzen tendenziell negative Arbeitsanreize für die betreuenden Elternteile und erzeugen Anreize gegen eine ausgewogene innerfamiliäre Arbeitsteilung. Im Vergleich zum modernisierten traditionellen Paradigma stellt das tendenziell einen gleichstellungspolitischen Rückschritt dar. Für diese Rückwendung zum traditionellen Paradigma mit einer starken Betonung innerfamiliärer Kinderbetreuung und -erziehung steht das Minus in der ersten Spalte der Tabelle 1 bei der FPÖ. Allerdings muss auch die FPÖ den gesellschaftlichen Entwicklungen Tribut zollen. Entgegen ihrer grundsätzlichen weltanschaulichen Festlegungen fordert sie aufgrund des ,immer stärker werdenden Wunsches nach besserer Vereinbarkeit von Beruf und Familie“ (FPÖ 2007: 81) ) $^{45}$ ein ausreichendes (vorzugsweise halbtägiges), qualitativ hochwertiges (außerfamiliäres) Kinderbetreuungsangebot bereitzustellen (FPÖ 2007: 86f.; 2009: 140ff.).

In den analysierten Positionspapieren der FPÖ wird das ökonomische Teilziel der optimalen Nutzung des Humanvermögens der Frauen/Mütter nicht direkt angesprochen. Vielmehr wird eine Verringerung bzw. Unterbrechung der Erwerbsarbeit des betreuenden Elternteils (in der Regel der Mutter) im Fall der Mutterschaft sogar explizit angestrebt. ${ }^{46}$ Diese hohe Bewertung der familiären Kindererziehung führt zu einer Reduktion des Arbeitskräftepotentials insbesondere der Frauen und auch zur potentiellen Entwertung ihres

\footnotetext{
44 Dazu wurden analysiert: das im Jahr 2005 beschlossene Parteiprogramm (FPÖ 2005) sowie das Handbuch freiheitlicher Politik (FPÖ 2007, 2009).

45 Vgl. ähnlich, aber etwas abgeschwächt FPÖ (2009: 143).

46 Implizit wird damit unterstellt, dass das Humanvermögen der Eltern (Frauen) in der Familie die bessere Wirkung entfaltet, als in der Erwerbsarbeit.
} 
Humanvermögens. ${ }^{47}$ Diese Akzeptanz des Verlusts von Humanvermögen der Mütter samt Nichtberücksichtigung der dargestellten ökonomischen Zielsetzungen ist als Rückwendung zum traditionellen Paradigma zu betrachten und führt wiederum zu einem Minus in der zweiten Spalte der Tabelle 1.

Analoges gilt für das Teilziel der Steigerung der Geburtenrate. Ihrer Erhöhung - vor allem mittels Familiensplitting - wird höchste Priorität zugemessen (FPÖ 2007: 81ff.; 2009: 131f.). ${ }^{48}$ Begründet wird dieses Ziel zwar auch mit der Sicherung der Sozialsysteme. Dies steht allerdings nicht im Vordergrund: Denn die Förderungen sollen auf - insbesondere kinderreiche - österreichische Familien beschränkt bleiben. Zudem wird Migration nicht als Lösung, sondern als Mitverursacher des Finanzierungsproblems gesehen, da die „Zuwanderung zu einem großen Teil in das Sozialsystem erfolgt ist“ (FPÖ 2009: 135). Schließlich wird betont, dass die (Mehr-)Kinderförderung bei österreichischen Familien letztlich eine „Frage des Überlebens unseres Volkes“ (FPÖ 2009: 137) sei. Wie oben gezeigt, wird schließlich eine Verbesserung der Vereinbarkeit von Erwerbsarbeit und Familie auf hohem Niveau mittels entsprechender Kinderbetreuungseinrichtungen etc. abgelehnt. All das läuft wieder auf eine Rückwendung zum traditionellen Paradigma hinaus und führt zum Minus in der dritten Spalte der Tabelle 1 bei der FPÖ.

In den analysierten Positionspapieren der FPÖ (2005: 29; 2007: 156; 2009: 230) werden nur wenige, sehr allgemein gehaltene Bezüge zwischen der Humanvermögensentwicklung von Kindern und Jugendlichen und den genannten ökonomischen Zielen hergestellt, explizit wird nur die Erhaltung der Wettbewerbsfähigkeit angeführt. In den Kapiteln zur Familienbzw. Bildungspolitik werden unter anderem folgende Forderungen erhoben: Laut FPÖ (2007: 155ff.; 2009: 229ff.) soll das Schulsystem weiterhin differenziert sein - eine gemeinsame Schule der 10- bis 14-Jährigen wird explizit abgelehnt (FPÖ 2005: 29; 2007: 155; 2009: 230); es soll stärker leistungsorientiert als derzeit sein, die Klassenschülerhöchstzahl soll 25 betragen. Weiters soll bei nachgewiesenen Sprachdefiziten von Kindern von Personen ohne österreichische Staatsbürgerschaft und ohne deutsche Muttersprache vor dem Schuleintritt „in gesonderten Kindergärten ein Intensivkurs absolviert [werden], der von den Eltern zu finanzieren ist" (2007: 29; 2009: 38). Nur bei bestandenem Sprachtest ist eine Aufnahme ins österreichische Schulsystem möglich. Zudem wird ein „verpflichtendes Vorschuljahr für Kinder mit nichtdeutscher Muttersprache, freiwillig für deutschsprachige Kinder" vorgeschlagen. ${ }^{49}$ Für KindergartenpädagogInnen wird - wie für alle PädagogInnen - ein tertiärer Abschluss gewünscht, indem die Berufsbildenden Höheren Schulen ,weiterentwickelt werden und zumindest einen Baccalaureatsabschluss anbieten“ (2007: 157; 2009: 231f.) sollen.

Zusammenfassend sind nur wenige Positionen erkennbar, die unter Berufung auf ökonomische Ziele und unter Verwendung humankapitaltheoretischer Argumente auf eine qualitativ hochwertige außerfamiliäre Ausbildung der Kinder und Jugendlichen auch schon in der frühkindlichen Lebensphase abzielen. Angesichts der schon besprochenen starken Präferenz für die innerfamiliäre Erziehung bzw. von Tagesmüttern/-vätern und des daraus resultierenden sehr verhaltenen Bekenntnisses für einen Ausbau der außerfamiliären (frühkindlichen) Kinderbetreuung ist das auch nicht weiter überraschend. Ein diesbezüglicher Paradigmenwechsel kann daher nicht diagnostiziert werden, deshalb das „,=“ in der vierten Spalte der Tabelle 1 bei der FPÖ.

Ziel des Bildungssystems ist die „Herstellung von Chancengerechtigkeit“ (FPÖ 2005: 28) ${ }^{50}$, wobei dieses ,jeder sozialen Schicht offenstehen muss“ (FPÖ 2007: 155; 2009: 229). Angesichts des beschriebenen weitgehenden Fehlens geeigneter Maßnahmen, die zu mehr Chancengleichheit in der und durch die Bildung beitragen können, werden die vorgeschlagenen Maßnahmen zum Abbau von Sprachdefiziten - trotz der grundsätzlich hohen Bedeutung dieses Teilthemas - für eine Überwindung des derzeitigen schichtspezifischen Bildungszugangs und eine Verbesserung sozialer Integration als nicht ausreichend eingeschätzt. Allenfalls sind sie aufgrund ihrer konkreten Ausgestaltung wie der starken Differenzierung zwischen Kindern mit deutscher und nichtdeutscher Muttersprache sogar kontraproduktiv. Deshalb ist bei der FPÖ auch bezüglich des Ziels der Chancengleichheit für Kinder kein Paradigmenwechsel erkennbar, wofür das ,,"“ in der letzten Spalte der Tabelle 1 steht.

\footnotetext{
47 Es wird aber auch die gegensätzliche Position vertreten, wonach in der Familienarbeit „Managerinnenkompetenzen" erworben werden, die bei einem Wiedereinstieg in die Erwerbsarbeit vorteilhaft sind.

48 Dass in FPÖ (2007: 82) noch explizit genannte Ziel von zwei Kindern pro Frau fehlt in FPÖ (2009).

49 Eine Kindergartenpflicht wird in FPÖ (2009: 144) explizit abgelehnt.

50 In FPÖ $(2007,2009)$ wird dieses Ziel nicht mehr genannt.
} 


\subsection{SPÖ: Vereinbarkeit von Erwerbsarbeit und Familie mittels qualitativ hochwertiger, außerfamiliärer Ganztagsbetreuung sowie ,, inklusiver" Schule}

Die SPÖ, die unter Bruno Kreisky in den 1970er Jahren noch weitreichende familienpolitische Änderungen, wie die Umstellung auf Individualbesteuerung, durchgesetzt hat, hat seit 1987 keine direkte Verantwortung für die Familienpolitik im Sinne einer Ressortverantwortlichkeit innegehabt.

Bezüglich des gleichstellungspolitischen Ziels gleicher Teilhabechancen von Männern und Frauen sind die familienpolitischen Positionen seit $1998^{51}$ durchgehend vom Ziel einer Verbesserung der Vereinbarung von Beruf und Familie geprägt, wobei sowohl eine innerfamiliär gerechtere Arbeitsteilung zwischen Vätern und Müttern als auch die eigenständige Absicherung von Frauen über die Zeit an Gewicht gewinnen. Neben einem Recht der Kinder auf „Betreuung inner- und außerhalb der Familie“ (SPÖ 1998: 17) werden unter anderem explizit gefordert: ein flächendeckender Ausbau der Ganztagsbetreuung für Kinder, insbesondere auch für unter 3-Jährige, der Übergang auf ganztägige Schulformen (SPÖ 2008: 16f.), Maßnahmen zur Erhöhung der Väterbeteiligung wie ein einkommensabhängiges Karenzgeld und ein „Papamonat“ (SPÖ 2008: 31; SPÖ-Frauen 2008: 7). Diverse Forderungen in SPÖ (2002) wie Rückkehrrechte nach dem Bezug des KBG auf einen Vollzeitarbeitsplatz oder in SPÖ-Frauen (2008: 6) nach einer qualifizierten Teilzeitarbeit sollen die Absicherung von Müttern verbessern. Diese sehr deutliche Abkehr vom modernisierten traditionellen Modell führt bei der SPÖ zu einem starken (fett gedruckten) gleichstellungspolitischen Doppelplus in der ersten Spalte der Tabelle 1.

Bezüglich der aus den obigen Maßnahmen resultierenden verstärkten Nutzung des $\mathrm{Hu}$ mankapitals der Frauen wird kein Paradigmenwechsel konstatiert, da sich dafür keine expliziten ökonomischen und humankapitaltheoretischen Argumente in den Positionspapieren finden lassen. Analoges gilt für das Teilziel einer Steigerung der Geburtenrate.

Die SPÖ weist dem Ausbau und der Qualitätssteigerung in den Bereichen außerfamiliärer Kinderbetreuung und Bildung eine sehr hohe Bedeutung zu und sieht diesbezügliche Aufgaben des Staates auch schon in der frühkindlichen Lebensphase. Dem „Kindergarten als Bildungseinrichtung“ wird in SPÖ (2004: 8) ein eigenständiger und bundeseinheitlicher Bildungsauftrag zugewiesen. In diesem „Bildungsgarten“ muss die „Lust am Lernen ... von Anfang an gefördert und unterstützt werden" (SPÖ 2008: 16). Eine Reihe von Maßnahmen soll neben den schon angesprochenen zu einer Verbesserung der frühkindlichen Bildung führen. Unter anderem wird für LehrerInnen und auch KindergartenpädagogInnen eine tertiäre Ausbildung angestrebt (SPÖ 2004: 9), die Schulpflicht soll auf das 5. Lebensjahr vorgezogen werden (SPÖ 2008: 17).

In SPÖ (1998: 21) wird Bildung ,als Chance der Entfaltung aller Fähigkeiten des Menschen und der Gesellschaft" beschrieben. Letzteres kann zwar als eine implizite ökonomische Begründung für die hohe Bedeutung einer guten Entwicklung des Humanvermögens von Kindern für die Gesellschaft interpretiert werden, in den analysierten Positionspapieren finden sich jedoch diesbezüglich - falls überhaupt - nur wenige direkte ökonomische Bezüge. Ähnlich wie bei der ÖVP verbleiben diese meist im Allgemeinen bzw. wird nur der individuelle Vorteil einer verbesserten Bildung erwähnt (SPÖ 1998: 21; 2004: 6f.; 2008: 12f., 16). Wegen dieser sehr vagen ökonomischen Begründungen wird, trotz der umfangreichen bildungsbezogenen Maßnahmenpakete in den Bereichen der Bildungs- und Familienpolitik, nur ein ansatzweise vorhandener Wechsel der Positionen diagnostiziert. Für die nur sehr schwache Hinwendung zu einem neuen Paradigma steht das eingeschränkte Plus in der vierten Spalte der Tabelle 1.

Die Ziele der Herstellung von Chancengleichheit in der und durch die Bildung werden dagegen mehrfach und deutlich formuliert (SPÖ 1998: 22; 2004: 6, 11f; 2008: 16). Durch die schon dargestellten Maßnahmen soll der schichtspezifische Zugang zur Bildung abgebaut werden. Außerfamiliäre Betreuungs- und Bildungseinrichtungen werden weiters laut SPÖ (2008: 17) als ,eines der wichtigsten Instrumente der Integration“ gesehen und sollen demgemäß gestärkt werden. Der Erwerb der deutschen Sprache und bei Kindern von

51 Analysiert wurden: Das 1998 beschlossene Grundsatzprogramm (SPÖ 1998), das Frauenprogramm 2002 (SPÖ 2002), das Bildungsprogramm 2004 (SPÖ 2004) sowie Wahlprogramme für die Nationalratswahl 2008 (SPÖ 2008, SPÖ-Frauen 2008). 
MigrantInnen auch der Muttersprache der Eltern wird dabei als besonders wichtig angesehen (SPÖ 2004: 8, 11; 2008: 17). Die in SPÖ (2004: 25ff.) geforderte ,inklusive“ Schule für alle Typen von Kindern mit besonderen Bedürfnissen soll auch dem Erwerb sozialer Kompetenzen dienen.

Obwohl weitere, die soziale Integration und den sozialen Zusammenhalt fördernde Elemente wie interkulturelles Lernen etc. in den analysierten Positionspapieren nicht explizit angesprochen werden, ist insgesamt gesehen eine sehr deutliche Hinwendung zu einem neuen Paradigma zu erkennen. Das wird mit einem (fett gedruckten) Doppelplus in der letzten Spalte der Tabelle 1 symbolisiert. Denn die SPÖ spricht sich eindeutig für eine Aufwertung auch der frühkindlichen außerfamiliären Betreuung und im Pflichtschulalter für gemeinsame, nur nach innen differenzierte, ganztägige Schulformen aus.

\subsection{Industriellenvereinigung und Sozialpartner: Gleichstellung ist notwendig, Vereinbarkeit ist ökonomisch sinnvoll, neue Schulen sind unverzichtbar}

Abschließend untersuchen wir die Einordnung der familienpolitischen Positionen der Industriellenvereinigung (IV) und der Sozialpartner. ${ }^{52}$ Die Positionen der IV sind seit 2004 im Wesentlichen unverändert und eindeutig ökonomisch begründet.

In einem gemeinsamen Positionspapier der IV mit den Sozialpartnern (2008: 1) wird die Vereinbarkeit von Erwerbsarbeit und Familie nicht nur zwecks Gleichstellung von Mann und Frau als gesellschaftspolitisch notwendig erachtet, sondern explizit auch als „ökonomisch sinnvoll“ bezeichnet. Denn „das qualitative und quantitative Potenzial der weiblichen Arbeitskräfte" werde unzureichend genutzt. Laut Brunner (2004) ist eine Nutzung des Humanvermögens der immer besser ausgebildeten Mütter angesichts der demographischen Entwicklung und des daraus resultierenden zukünftigen Arbeitskräftemangels zur Erhaltung der internationalen Wettbewerbsfähigkeit Österreichs in der sich abzeichnenden Wissensgesellschaft erforderlich. ${ }^{53}$ Zur Absicherung des Wirtschaftswachstums wird zusätzlich eine Zuwanderungspolitik als notwendig erachtet, wofür jedoch ein fremden- und integrationsfreundliches Klima geschaffen werden muss.

Gemeinsam mit der Arbeiterkammer (Brunner 2005), später mit den Sozialpartnern (2008) und schließlich in IV (2009) wurden zu diesem Zweck umfassende Maßnahmenkataloge erstellt. Diese beinhalten unter anderem: einen breiten quantitativen und qualitativen, an die beruflichen Erfordernisse angepassten Ausbau der Kinderbetreuungseinrichtungen insbesondere auch für unter 3-Jährige, ${ }^{54}$ um einen frühzeitigeren Wiedereinstieg in den Beruf zu ermöglichen; eine Flexibilisierung des KBG zwecks früherer Rückkehr in den Arbeitsprozess, Maßnahmen zur Erhöhung der Väterbeteiligung in der Kindererziehung, besser an die familiäre Situation angepasste innerbetriebliche Gestaltung der Arbeitsplätze und Karrierepfade; Modelle von Work-Life-Balance, die auch Bildungskarenzen etc. einschließen. Zusätzlich zu Verbesserungen bei der außerfamiliären Kinderbetreuung wird eine schon im Kindergarten einsetzende Bewusstseinsbildung, die ,,veraltete Rollenbilder schnell und nachhaltig“ (IV 2009: 5) verändert, für unverzichtbar gehalten.

Gleichstellungspolitisch spricht all das für eine klare Abkehr vom modernisierten traditionellen Familienmodell. Diese deutliche Hinwendung zu einem neuen Paradigma lässt ein Doppelplus in der ersten Spalte der Tabelle 1 bei der IV samt Sozialpartnern für gerechtfertigt erscheinen, wenngleich die IV in diesem Bereich gesetzliche Regulierungen wie Quoten oder auch ein Recht auf Elternteilzeit, die aber ausgebaut werden soll, ablehnt. Vielmehr werden die betroffenen Institutionen aufgerufen, „gemeinsame, freiwillige Lösungen“ (IV 2009: 9) zu suchen.

Ausgenommen das Thema der Sicherung der Sozialsysteme werden die im Abschnitt 3 besprochenen ökonomischen Argumentationslinien zugunsten einer verstärkten quantitativen und qualitativen Nutzung des Humanvermögens der Frauen und Mütter in den

52 Die folgende Beurteilung stützt sich auf diverse Positionspapiere der IV (2007, 2009, 2010), Brunner (2004) sowie auf gemeinsame Positionspapiere der IV mit der Arbeiterkammer (Brunner 2005) bzw. den Sozialpartnern (Sozialpartner 2008), das sind die Arbeiter-, die Landwirtschafts- und die Wirtschaftskammer Österreich sowie der Österreichische Gewerkschaftsbund. Der Zeitraum vor 2004 wurde nicht analysiert.

53 Auch in einem jüngeren Positionspapier der IV (2009) werden entsprechende Argumente verwendet.

54 Dazu zählen unter anderem: mehr Betreuungsplätze insbesondere für unter 3-Jährige, Nachmittagsbetreuung für Schulkinder zwischen 6 und 14 Jahren, Anpassung der täglichen Öffnungs- und jährlichen Schließzeiten an die beruflichen Erfordernisse, einheitliche Qualitätsstandards für Ausbildung und Kinderbetreuung. 
untersuchten Positionspapieren der IV und der Sozialpartner wie oben gezeigt durchgehend aufgenommen. Diese sehr deutliche Hinwendung zu einem neuen Paradigma rechtfertigt das starke (fett gedruckte) Doppelplus in der zweiten Spalte der Tabelle 1.

Die Steigerung der Geburtenrate wird in den analysierten Positionspapieren nicht explizit als Ziel genannt. Laut IV (2009: 7) erleichtert jedoch eine Verbesserung der Vereinbarkeit von Familie und Beruf die Realisierung der Kinderwünsche und trägt damit indirekt zur „Entschärfung der demografischen Entwicklung“ bei - womit der mittelfristig erwartete Mangel an (qualifizierten) Arbeitskräften angesprochen wird. Ein positiver Einfluss einer hohen Erwerbsbeteiligung von Frauen auf die Geburtenrate wird nicht postuliert. ${ }^{55}$ Diesbezüglich liegt also kein Paradigmenwechsel laut Definition vor, wofür das „,=“ in der dritten Spalte der Tabelle 1 steht.

Die hohe Bedeutung einer guten Humanvermögensentwicklung von Kindern und Jugendlichen in den Kindergärten und an den Schulen für den Wirtschaftsstandort Österreich und seine zukünftige Entwicklung, die alle Begabungen und Talente bestmöglich fördert, wird in diversen Papieren der IV $(2007,2010)$ klar hervorgehoben. In der 2006 verabschiedeten Vision „Schule 2020“ werden Maßnahmen für eine umfassende Neugestaltung des Bildungswesens in Österreich vorgeschlagen. Laut IV (2007: 5; im Original zum Teil fett) sind „Familie, frühkindliche Entwicklung, Kindergarten und Elementarbildung ... die wichtigsten Grundlagen für eine erfolgreiche Bildungslaufbahn und gelingende Lebenswege." Doch aufgrund ,verschiedenartig geänderter sozialer Hintergründe können viele Elternteile ..., können familiäre Umgebung und sozialer Rahmen diese Aufgabe nicht mehr ausreichend erfüllen.“ Deshalb muss das „Schulwesen ... für Kinder und Jugendliche zunehmend dem veränderten gesellschaftlichen und sozialen Umfeld als ,Heim der zweiten Stufe' umfassend Rechnung tragen.“ Diese „Lebensschule“ muss zu einem umfassenden Kompetenzzentrum für lebenslanges Lernen ausgebaut werden und soll für die SchülerInnen zu einem jugend- und altersgerechten Lebensraum werden, in dem sie sprachliche, fachliche, soziale und emotionale Kompetenzen festigen und erwerben (IV 2010: 23ff.). Um diese Ziele zu verwirklichen, werden unter anderem folgende konkrete Maßnahmen vorgeschlagen: ein verpflichtendes, kostenloses Startschuljahr ab dem 5. Lebensjahr, an die Berufs- und Lebenswelt der Eltern angepasste ganztägige Schulformen, Entwicklung der (fremd)sprachlichen Kompetenzen bereits ab dem Kindergarten auch durch das Erlernen der Muttersprache als Grundlage für das Erlernen von Deutsch - bei Kindern von MigrantInnen - sowie eine frühe Förderung von Englisch, mehr männliche Pädagogen und PädagogInnen mit Migrationshintergrund, kleinere Kindergartengruppen (IV 2009: 11ff.; 2010: 25ff.). „Kinder mit besonderem kulturellen, sprachlichen oder mit sozial belastetem Hintergrund sind bereits in frühesten Bildungsphasen durch dafür besonders ausgebildete Fachkräfte“ (IV 2007: 8; im Original zum Teil fett) gezielt zu unterstützen.

Diese Ziele und Maßnahmenvorschläge werden als ein sehr deutlicher, ökonomisch begründeter bildungspolitischer Paradigmenwechsel eingestuft, wofür das fette Doppelplus in der vorletzten Spalte der Tabelle 1 bei der IV und den Sozialpartner steht. In den dargestellten Positionen sind auch diverse integrationsfördernde und den sozialen Zusammenhalt stärkende Elemente enthalten, doch da die Herstellung von Chancengleichheit für Kinder - im Unterschied zur SPÖ - nicht explizit im Vordergrund steht, wird „,nur“ eine deutliche Hinwendung zu einem neuen Paradigma diagnostiziert und in der letzten Spalte der Tabelle 1 mit einem Doppelplus gekennzeichnet.

\section{Zum Paradigmenwechsel in familienpolitischen Positionen}

In diesem abschließenden Abschnitt wird eine zusammenfassende Einordnung der Entwicklung der familienpolitischen Positionen der untersuchten politischen Parteien und Interessenvertretungen in Österreich in den vergangenen 15 Jahren vorgenommen.

Aus gleichstellungspolitischer Perspektive spielt das Thema der Erleichterung bzw. Verbesserung der Vereinbarkeit von Erwerbsarbeit und Familie bei allen analysierten Institutionen vordergründig eine wichtige Rolle. Ein Blick hinter die Fassade zeigt jedoch, dass sich die jeweiligen Positionen im Detail doch deutlich unterscheiden, wie auch aus den sehr unterschiedlichen Qualifizierungen der jeweiligen Positionen in der ersten Spalte der

55 Es wird nur darauf hingewiesen, dass ausreichende und vielfältige Kinderbetreuungseinrichtungen die Fertilitätsrate positiv beeinflussen (IV 2009: 14). 
Tabelle 1 ersichtlich wird. Bei der FPÖ erscheint die Befürwortung vereinbarkeitsfördernder Kinderbetreuungseinrichtungen eher nur als eine Konzession an den Zeitgeist, grundsätzlich wird ein tendenziell stark traditionelles Familienmodell vertreten.

In abgeschwächter Form gilt, das auch für die ÖVP, deren Position im Vergleich zur FPÖ aber sowohl „vielschichtiger“ als auch widersprüchlicher ist. Sie betont einerseits die Familie als Keimzelle der Gesellschaft und propagiert andererseits die individuelle Wahlfreiheit, was auch Entscheidungen gegen traditionelle Familienformen möglich erscheinen lässt. Die ÖVP fordert Chancengleichheit für die Kinder, spricht sich aber auch gegen Maßnahmen aus, die diese Chancengleichheit fördern usw.

Bezüglich der Forderung nach Erhalt und Ausbau von Wahlfreiheit zeigt sich bei genauerem Blick zum einen, dass diese vielfach nur eine formale ist. Beispielsweise wird kein rascher, flächendeckender Ausbau der Kinderbetreuungseinrichtungen insbesondere für Kleinkinder unter drei Jahren gefordert. Auch die Nachmittagsbetreuung für 6- bis 14-Jährige wird nicht wirklich forciert. Da die außerfamiliäre Kinderbetreuung im ÖVP-Parteiprogramm 1995 und auch im Bildungskonzept 2011 weiterhin grundsätzlich als subsidiär zur Betreuung in der Familie angesehen wird, ist dieses Ergebnis nicht wirklich überraschend. Wenn die besprochenen Mittel zur Ausübung der Wahlfreiheit aber nicht in ausreichendem Ausmaß verfügbar sind, wird diese für die betroffenen Eltern zu einer substanzlosen Floskel, die zwar einen formalen Anspruch postuliert, der aber faktisch (ohne Inkaufnahme von grundsätzlich vermeidbaren Nachteilen) nicht einlösbar ist. ${ }^{56}$

Zum anderen ist eine politisch neutrale Familienpolitik eine aus theoretischen und praktischen Gründen nicht verwirklichbare Illusion und kein geeignetes Leitbild für eine moderne Familienpolitik, wie Sturn in seinem Beitrag in diesem Band im Detail ausführt. Praktisch gesehen, dürfte eine staatliche Stützung für einen Parallelbetrieb aller möglichen Typen von Betreuungsregimes an den damit verbundenen hohen Kosten scheitern. Doch auch wenn dies als möglich unterstellt wird, kann auf diesem Weg aus theoretischen Gründen keine vollständige Neutralität erzielt werden. Das staatlich gestützte Nebeneinander unterschiedlicher Typen von Betreuungsstrukturen ist nicht neutral bezüglich der relativen Attraktivität dieser Alternativen zueinander. Veränderungen in den relativen Kosten bzw. relativen Vorteilen der zur Wahl stehenden Alternativen erzeugen ökonomische Anreize für bzw. gegen einzelne Alternativen. Diese Anreize beeinflussen die Auswahl auf Grundlage der jeweils individuellen Präferenzen. Solche Anreizeffekte entstehen auch dann, wenn FamilienpolitikerInnen vorgeben, sie nicht auslösen zu wollen und können nicht wegdiskutiert werden. Diskutabel ist natürlich die Stärke dieser Anreize. Diese ist aber von der - durch die staatliche Unterstützung beeinflussten - relativen Attraktivität der Alternativen zueinander abhängig. Familienpolitik kann deshalb nicht vollständig neutral sein. Zudem kann ein solcher Parallelbetrieb unterschiedlicher Betreuungsstrukturen zu Koordinationsproblemen und vermeidbaren innerfamiliären und gesellschaftlichen Koordinationskosten führen. Die von der ÖVP propagierte neutrale staatliche Familienpolitik, die vorgibt, nur Wahlmöglichkeiten offen zu halten bzw. neue Optionen zu öffnen und ansonsten völlig neutral zu sein, ist deshalb nicht möglich bzw. ökonomisch nicht wünschenswert.

Zusammenfassend wird in den ÖVP-Papieren eine modernisierte Variante des traditionellen breadwinner-Modells sichtbar, bei der die Vereinbarkeit auf nicht existenzsichernden Kombinationen von Teilzeitarbeit und zeitweise vollem Ausstieg aus der Erwerbsarbeit vor allem für Frauen beruht und bei der die außerhäusliche Kinderbetreuung grundsätzlich eher die Ausnahme bilden und nur subsidiär eingesetzt werden soll. Somit ist bei FPÖ und ÖVP kein grundlegender gleichstellungspolitischer Paradigmenwechsel erkennbar.

Dagegen ergibt sich aus den Positionspapieren der SPÖ und der IV mit den Sozialpartnern eine starke Hinwendung zu einem Paradigma einer umfassenden Geschlechtergleichstellung. Es wird ein breiter Ausbau qualitativ hochwertiger außerhäuslicher Kinderbetreuungseinrichtungen auch schon für Kleinkinder vorgeschlagen. Während die SPÖ diese Forderungen vor allem gleichstellungspolitisch begründet, stehen bei der IV und den Sozialpartnern die ökonomischen Argumente im Vordergrund. Deshalb wird bei der SPÖ ein vergleichsweise stärkerer gleichstellungspolitischer Paradigmenwechsel diagnostiziert.

Wie in der ersten Spalte der Tabelle 1 ersichtlich, sind die gleichstellungspolitischen Positionen der untersuchten Akteure sehr heterogen. Sie erstrecken sich von einer

56 Dazu kommt, dass die in den Programmen ursprünglich nicht vorgesehene, dann aber gemeinsam mit der FPÖ beschlossene Form des Kinderbetreuungsgeldes einen starken negativen Anreiz für einen frühen Berufseinstieg nach der Babypause bot und damit bezüglich der Erwerbsbeteiligung von Frauen kontraproduktiv wirkte. 
Rückwendung zum traditionellen Familienmodell bis zu einer (sehr) deutlichen Hinwendung zu einem neuen Paradigma, wobei die Mittelposition vollständig fehlt. Das gilt analog auch für andere der untersuchten Ziele.

Die gesamtwirtschaftlichen Ziele der Sicherung von Produktivität und Wirtschaftswachstum werden in den untersuchten Positionspapieren der politischen Parteien im Zusammenhang mit der Familien- und Bildungspolitik - falls überhaupt - nur sehr allgemein angesprochen. Das Thema der Sicherung der Finanzierung der Sozialsysteme findet sich in diesen Zusammenhängen überraschenderweise überhaupt nicht.

Auch das damit verknüpfte, in der zweiten Spalte dargestellte Teilziel einer umfassenden Nutzung des Humanvermögens der zunehmend gut ausgebildeten Frauen und Mütter wird von den politischen Parteien nicht explizit verwendet. Viele der in den Positionspapieren der SPÖ geforderten Maßnahmen zur Verbesserung der Vereinbarkeit von Erwerbsarbeit und Familie der SPÖ begünstigen zwar die Ausschöpfung des weiblichen Humanvermögens, doch sie werden durchgehend nur gleichstellungspolitisch begründet. Dagegen wählen die Verbände den drohenden zukünftigen Arbeitskräftemangel und die Erhaltung von Wettbewerbsfähigkeit und Wachstum als Ausgangspunkt für die Begründung vielfach in die gleiche Richtung laufender Forderungen. Auch bezüglich dieses Ziels ergeben sich deutliche Unterschiede in den Positionen der analysierten Institutionen.

Das Ziel einer Steigerung der Geburtenrate wird vor allem in den Papieren von ÖVP und FPÖ formuliert. Für die FPÖ ist dies ein Kernthema, für die SPÖ dagegen eher ein Randthema, die IV behandelt es nur indirekt. Keiner der Akteure nimmt die empirisch gestützte Argumentation auf - diesbezüglich gibt es wohl die größte Übereinstimmung -, wonach es zu einer Steigerung der Fertilität unter den heutigen Rahmenbedingungen notwendig und förderlich sein könnte, den Müttern ein hohes Ausmaß an Teilhabe am Arbeitsmarkt zu ermöglichen. Vielmehr wird insbesondere von der FPÖ auf Konzepte von Gestern gesetzt. Demnach muss es einem Elternteil ermöglicht werden, sich zur Betreuungsarbeit aus der Erwerbsarbeit zurückzuziehen. Tendenziell läuft das auf eine Rückwendung zu einem Familienmodell mit traditioneller Arbeitsteilung zwischen Mann und Frau hinaus.

Bezüglich der Humanvermögensentwicklung der Kinder lassen sich ebenfalls keine einheitlichen Positionen ausmachen. Ein durchgehender Paradigmenwechsel, der als Ausbau außerfamiliärer Kinderbetreuungseinrichtungen mit hoch qualifiziertem Betreuungspersonal, die ganztägig und teils verpflichtend zu besuchen sind und auch schon im frühkindlichen Alter einsetzen, definiert ist, kann nicht festgestellt werden, wie in den beiden letzten Spalten der Tabelle 1 ersichtlich wird.

In den Positionspapieren von ÖVP und SPÖ wird zwar ansatzweise auf die individuellen ökonomischen Vorteile verbesserter Bildung hingewiesen. Doch auch in diesem Zusammenhang werden humankapital- und wachstumstheoretische Bezüge nur sehr vage und meist nur implizit hergestellt. Nur die IV bezieht sich klar auf gesamtwirtschaftliche Aspekte wie Wettbewerbsfähigkeit, Wohlstand und Wachstum. Die entsprechenden bildungspolitischen Forderungen in den ÖVP-Papieren bleiben deutlich hinter jenen in den untersuchten Positionspapieren der SPÖ und der IV zurück. Die FPÖ misst insbesondere im Kindergartenalter der Erziehung im Familienverband einen noch höheren Stellenwert zu als die ÖVP.

Die Herstellung von Chancengleichheit in der und durch Bildung samt der potentiell positiven Funktion von Bildung für die soziale Integration und den gesellschaftlichen Zusammenhalt werden zwar von allen politischen Parteien thematisiert. Im Detail unterscheiden sich die Positionen und die daraus abgeleiteten Forderungen aber wieder deutlich. Die integrative Funktion von Bildung wird insbesondere von der SPÖ - etwas schwächer ausgeprägt - auch von der IV vertreten. Es folgen mit deutlichem Abstand die ÖVP und dann die FPÖ. Die SPÖ betont in diesem Zusammenhang das Ziel des Abbaus des vorhandenen schichtspezifischen Bildungszugangs durch die Schaffung unter anderem gemeinsamer, in sich differenzierender, inklusiver Schulen. Von der IV wird die Herstellung von Chancengleichheit nicht direkt als Ziel formuliert, wenngleich viele der vorgeschlagenen Maßnahmen wie individuell differenzierende Förderung, die auch auf besondere persönliche Umstände Bedacht nimmt, in diese Richtung wirken. Für die 10- bis 14-Jährigen wird eine ,leistungsorientierte Differenzierung bei einem gemeinsamen, vergleichbaren Bildungsangebot" (IV 2010: 25) gefordert.

Es ist auffallend, dass die SPÖ in den beiden gerechtigkeitsbezogenen Bereichen (gleiche Teilhabechancen von Frauen und Männern am Arbeitsmarkt und in der Familie, 
Chancengleichheit für die Kinder im Bildungsbereich) jeweils eindeutig und sehr deutlich für einen Paradigmenwechsel eintritt. Dagegen wird das Argumentationspotential für entsprechende Veränderungen in den effizienzbezogenen, ökonomischen Bereichen bei weitem nicht ausgeschöpft. ${ }^{57}$

Dazu spiegelbildlich ist die Position der Verbände, welche die ökonomischen Ziele (erwartungsgemäß) relativ stärker hervorheben. Es ist auch auffallend, dass die Position der IV bezüglich der Erwerbsbeteiligung der Frauen und der Geschlechtergleichstellung wie auch bezüglich der Humanvermögensentwicklung der Kinder jener der SPÖ viel näher ist, als jener der ÖVP. Die Übernahme der stark ökonomisch geprägten Positionen der IV, die einer Abwendung vom modernisierten traditionellen Paradigma gleichkommt, scheitert in der ÖVP offensichtlich an starken Gegenkräften. ${ }^{58}$

Zusammenfassend haben die eingangs angeführten vielfältigen Veränderungen von Rahmenbedingungen und sich daraus ergebenden Probleme noch zu keiner eindeutigen und breit getragenen Herausbildung neuer Paradigmen geführt. Die familien- und bildungspolitischen Positionen der entscheidungsrelevanten politischen Akteure divergieren weiterhin deutlich.

\section{Literatur}

Bock-Schappelwein, Julia/Eppel, Rainer/Mühlberger, Ulrike (2009): Sozialpolitik als Produktivkraft. Österreichisches Institut für Wirtschaftsforschung. April 2009. Wien.

BMWFJ - Bundesministerium für Wirtschaft, Familie und Jugend (2010): 5. Familienbericht 1999 2009. Die Familie an der Wende zum 21. Jahrhundert. Band I und II. Wien.

Brunner, Judith (2004): Zukunft der Beschäftigung. IV-Positionen April 2004: 6. www.iv-mitglieder service.at/iv-all/dokumente/doc_2069.pdf (02.03.2011).

Brunner, Judith (2005): IV und AK: Vereinbarkeit von Familie und Beruf als Investition in die Zukunft. 08.11.2005. www.iv-net.at/b1500m120 (02.03.2011).

Die Presse (2008): Wahlprogramm: ÖVP setzt auf Sicherheit und Familie. 01.09.2008. diepresse.com /home/politik/neuwahlen/410563 (02.03.2011).

Die Presse (2009). ÖVP gibt Startschuss für neues Parteiprogramm. 25.02.2009. diepresse.com/home /politik/innenpolitik/455618 (02.03.2011).

Dingeldey, Irene (2000) (Hrsg.): Erwerbstätigkeit und Familien in Steuer- und Sozialversicherungssystemen. Begünstigungen und Belastungen verschiedener familialer Erwerbsmuster im Ländervergleich. Opladen: Leske + Budrich.

Dujmovits, Rudolf (2009): Zur Entwicklung familienpolitischer Positionen ausgewählter politischer Parteien und Interessenvertretungen in Österreich. Working Paper No. 2009-3. Institut für Finanzwissenschaft und Öffentliche Wirtschaft. Dezember 2009. Universität Graz.

Evers, Adalbert/Heinze, Rolf G. (Hrsg.) (2008): Sozialpolitik. Ökonomisierung und Entgrenzung. Wiesbaden: VS Verlag für Sozialwissenschaften.

FPÖ (2005): Das Parteiprogramm der Freiheitlichen Partei Österreichs. Mit Berücksichtigung der beschlossenen Änderungen vom 27. Ordentlichen Bundesparteitag der FPÖ am 23. April 2005 in Salzburg. Www.fpoe.at/fileadmin/Content/portal/PDFs/2009/fp_parteiprogramm_neu.pdf (02.03.2011).

FPÖ (2007): Handbuch freiheitlicher Politik. Ein Leitfaden für Führungsfunktionäre und Mandatsträger der Freiheitlichen Partei Österreichs. Wien.

FPÖ (2009): Handbuch freiheitlicher Politik. Ein Leitfaden für Führungsfunktionäre und Mandatsträger der Freiheitlichen Partei Österreichs. 2. Auflage. Wien. www.fpoe.at/fileadmin/Content/portal/PDFs/2009/Handbuch_FPOE_webok.pdf (02.03.2011).

Fuchs, Michael (2006): Kinderbetreuungsplätze in Österreich. „Fehlen keine oder bis zu 650.000?“ Bedarfsanalysen 2005 - 2015 im Auftrag der Industriellenvereinigung. Europäisches Zentrum für Wohlfahrtspolitik und Sozialforschung. Wien.

Geisberger, Tamara/Knittler, Käthe (2010): Niedriglöhne und atypische Beschäftigung in Österreich. Statistische Nachrichten 2010 (6): 448-461.

Hall, Peter A. (1993): Policy Paradigms, Social Learning, and the State. Comparative Politics 25 (3): 239-255.

57 Für das Thema Bildung mag das Verständnis von Bildung als „Wert an sich“ (SPÖ 2008: 16) diese Ausblendung ökonomischer Argumente ein Stück weit verständlich machen.

58 Erwähnenswert ist in diesem Zusammenhang, dass die österreichische Familienpolitik seit Anfang Dezember 2008 dem Wirtschaftsministerium zugeordnet ist, welches in Bundesministerium für Wirtschaft, Familie und Jugend umbenannt worden ist. Für die familienpolitischen Agenden ist eine Staatssekretärin zuständig. 
Held, Martin/Kubon-Gilke, Gisela/Sturn, Richard (Hrsg.) (2009): Bildungsökonomie in der Wissensgesellschaft. Jahrbuch Normative und institutionelle Grundfragen der Ökonomik. Band 8. Marburg: Metropolis.

Hink, Robert (2009): Ablehnung vom Gemeindebund. Dann droht eine Kostenexplosion. Kommunal 2009 (4): 19.

IV - Industriellenvereinigung (2007): Zukunft der Bildung - Lehrerinnen \& Lehrer. Mai 2007. Wien. iv-mitgliederservice.at/iv-all/publikationen/file_396.pdf (02.03.2011).

IV (2009): Gleiche Chancen. Bessere Vereinbarkeit. Gleichstellung von Frauen und Männern. Vereinbarkeit von Familie und Beruf. November 2009. Wien. iv-mitgliederservice.at/iv-all /publikationen/file_514.pdf (02.03.2011).

IV (2010): Schule 2020: Lernen - Wachstum - Wohlstand. Zwischenbilanz. 2. aktualisierte Auflage. Februar 2010. Wien. iv-mitgliederservice.at/iv-all/publikationen/file_503.pdf (02.03.2011).

Jacob, Marita (2009): Der Einfluss des Elternhauses auf dem Weg zum Hochschulabschluss: Soziale Ungleichheit im Bildungsverlauf. In: Held/Kubon-Gilke/Sturn (2009): 169-191.

Kalina, Thorsten/Weinkopf, Claudia (2008): Konzentriert sich die steigende Niedriglohnbeschäftigung in Deutschland auf atypisch Beschäftigte? Zeitschrift für Arbeitsmarktforschung 41 (4): 447-469.

Klenner, Christina (2007): Gleichstellungspolitik vor alten und neuen Herausforderungen - Welchen Beitrag leistet die Familienpolitik? WSI Mitteilungen 60 (10): 523-530.

Leitner, Sigrid (2003): Varieties of Familialism. The Caring Function of the Family in Comparative Perspective. European Societies 5 (4): 353-375.

Leitner, Sigrid (2008): Ökonomische Funktionalität der Familienpolitik oder familienpolitische Funktionalisierung der Ökonomie? In: Evers/Heinze (2008): 67-82.

Lewis, Jane/Ostner, Ilona (1994): Gender and the Evolution of European Social Policies. ZeS-Arbeitspapier Nr. 4/94. Universität Bremen.

Meier, Isabella/Kreimer, Margareta (2011): „Die Angehörigen wissen am besten was gut ist“. Eine Analyse des Systems der familiären Langzeitpflege und dessen Auswirkungen auf die Lage pflegender Angehöriger. Graz: Grazer Universitätsverlag.

Mikl-Leitner, Johanna (2007): Ideen. Impulse. Ziele. Die ÖVP denkt vor: Familie und Kinder. Perspektivengruppe 2010. Impulsgruppe Familie und Kinder. Ergebnisse des Diskussionsprozesses unter der Leitung von LR Mag. Johanna Mikl-Leitner. 26. September 2007. www.oevp.at /common/downloads/P2010_FamilieundKinder_Gesamt.pdf (02.03.2011).

Musiol, Daniela/Fellerer, Anja (o. J.): Frühförderung im Kindergarten. www.gruene.at/bildung_jugend/fruehfoerderung (02.03.2011).

Ostner, Ilona (2006): Paradigmenwechsel in der (west)deutschen Familienpolitik. In: Berger, Peter A./Kahlert, Heike (Hrsg.): Der demographische Wandel: Chancen für die Neuordnung der Geschlechterverhältnisse. Frankfurt a. M.: Campus: 165-199.

Ostner, Ilona (2008): Ökonomisierung der Lebenswelt durch aktivierende Familienpolitik? In: Evers/Heinze (2008): 49-66.

ORF (2011): Karl: Eltern in die Pflicht nehmen. Ministerin Karl im Gespräch mit Wolfgang Werth. Morgenjournal 10.01.2011. oe1.orf.at/artikel/266706 (02.03.2011)

ÖVP (1998): Grundsatzprogramm. Beschlossen am 30. ordentlichen Parteitag der Österreichischen Volkspartei am 22. April 1995 in Wien. www.oevp.at/download/000298.pdf (02.03.2011).

ÖVP (2007): Perspektiven für Österreich - Perspektiven für die Menschen. Die ÖVP denkt vor: Perspektiven 2010. Ideen. Impulse. Ziele. Wien.

ÖVP (2008): Neustart für Österreich. Wahlprogramm der ÖVP für die Nationalratswahl 2008. www. oevp.at/Common/Downloads/Wahlprogramm_innen_Ansicht.pdf (02.03.2011).

ÖVP (2011): Unser Bildungsweg für Österreich. Jänner 2011. www.oevp.at/download /1117114931022626.pdf (02.03.2011).

ÖVP-Frauen (2008): Das ÖVP-Frauen Programm für die Nationalratswahl 2008. www.frauenoffen sive.at/Common/Downloads/Programm.pdf (02.03.2011).

Sarrazin, Thilo (2010): Deutschland schafft sich ab. München: DVA.

Sozialpartner (2008): Gemeinsames Grundsatzpapier der Sozialpartner und der Industriellenvereinigung „Gleichstellung von Frauen und Männern als Anliegen der Interessenvertretungen der ArbeitnehmerInnen und ArbeitgeberInnen“. 30.10.2008. Wien. www.iv-mitgliederservice.at/ivall/dokumente/doc_2787.pdf (02.03.2011).

SPÖ (1998): Das Grundsatzprogramm. Wien: SPÖ-Bundesgeschäftsstelle. www.spoe.at/bilder/d251 /spoe_partei_programm.pdf (02.03.2011).

SPÖ (2002): Faire Chancen für alle Frauen. Das 10-Punkte-Programm der SPÖ. ireds.spoe.at/bilder /PK_Sozialstaat_frauen.pdf (02.03.2011).

SPÖ (2004): Das Bildungsprogramm der SPÖ. Bildung - Grundlage unseres Lebens, beschlossen am 38. Ordentlichen Bundesparteitag, 29. und 30. November 2004. www.spoe.at/bilder/d251/bildung_web.pdf (02.03.2011).

SPÖ (2008): Wahlmanifest der Sozialdemokratischen Partei Österreichs. Nationalratswahl 2008, 40. Ordentlicher Bundesparteitag 8. August 2008, Design Center Linz. Wien: SPÖ-Bundesgeschäftsstelle. 
SPÖ-Frauen (2008): Wahlprogramm SPÖ Frauen 2008: Mit neuer Kraft! Für mehr Chancen, Rechte und Einkommen. www.erhoert.at/SPOE_Wahlprogramm_Frauen_spoe_Letzfassung20080805 mh.pdf (02.03.2011).

Statistik Austria (2010): Bildung in Zahlen 2008/2009. Wien.

Steinmayr, Andreas (2009): Die Bildungssituation der zweiten Zuwanderergeneration in Wien. ÖIFDossier Nr. 3. Österreichischer Integrationsfonds. Wien.

Wößmann, Ludger (2009): Beeinflusst Bildungsselektion Bildungsergebnisse und Ungleichheit? Internationale und nationale Evidenz. In: Held/Kubon-Gilke/Sturn (2009): 147-167. 